\title{
Lymphatic vessels regulate immune microenvironments in human and murine melanoma
}

\author{
Amanda W. Lund, ${ }^{1,2}$ Marek Wagner, ${ }^{3,4}$ Manuel Fankhauser, ${ }^{2}$ Eli S. Steinskog, ${ }^{3}$ Maria A. Broggi, ${ }^{2}$ Stefani Spranger, ${ }^{5}$ \\ Thomas F. Gajewski, ${ }^{5,6}$ Kari Alitalo, ${ }^{7}$ Hans P. Eikesdal, ${ }^{8,9}$ Helge Wiig, ${ }^{3}$ and Melody A. Swartz ${ }^{2,10}$ \\ 'Department of Cell, Developmental and Cancer Biology and Knight Cancer Institute, Oregon Health and Science University, Portland, Oregon, USA. ${ }^{2}$ Institute of Bioengineering and Swiss Institute \\ of Experimental Cancer Research (ISREC), Ecole Polytechnique Federale de Lausanne, Lausanne, Switzerland. ${ }^{3}$ Department of Biomedicine, University of Bergen, Bergen, Norway. ${ }^{4}$ Department of Pathology, \\ Haukeland University Hospital, Bergen, Norway. ${ }^{5}$ Department of Pathology and ${ }^{6}$ Department of Medicine, University of Chicago, Chicago, Illinois, USA. ${ }^{7}$ Wihuri Research Institute and Translational Cancer \\ Biology Program, Biomedicum Helsinki, University of Helsinki, Helsinki, Finland. ${ }^{8}$ Section of Oncology, Department of Clinical Science, University of Bergen, Bergen, Norway. ${ }^{9}$ Department of Oncology, \\ Haukeland University Hospital, Bergen, Norway. ${ }^{10}$ Institute for Molecular Engineering, University of Chicago, Chicago, Illinois, USA.
}

Lymphatic remodeling in tumor microenvironments correlates with progression and metastasis, and local lymphatic vessels play complex and poorly understood roles in tumor immunity. Tumor lymphangiogenesis is associated with increased immune suppression, yet lymphatic vessels are required for fluid drainage and immune cell trafficking to lymph nodes, where adaptive immune responses are mounted. Here, we examined the contribution of lymphatic drainage to tumor inflammation and immunity using a mouse model that lacks dermal lymphatic vessels (K14-VEGFR3-Ig mice). Melanomas implanted in these mice grew robustly, but exhibited drastically reduced cytokine expression and leukocyte infiltration compared with those implanted in control animals. In the absence of local immune suppression, transferred cytotoxic $T$ cells more effectively controlled tumors in K14-VEGFR3-Ig mice than in control mice. Furthermore, gene expression analysis of human melanoma samples revealed that patient immune parameters are markedly stratified by levels of lymphatic markers. This work suggests that the establishment of tumor-associated inflammation and immunity critically depends on lymphatic vessel remodeling and drainage. Moreover, these results have implications for immunotherapies, the efficacies of which are regulated by the tumor immune microenvironment.

\section{Introduction}

The aberrant growth of regional lymphatic vessels - often referred to as lymphangiogenesis or lymphatic hyperplasia - is associated with enhanced locoregional metastasis and poor outcome in many solid tumors including melanoma (1). Lymphatic vessels contribute to tumor progression at least in part by actively facilitating metastatic dissemination to sentinel lymph nodes through a variety of mechanisms, both in primary tumors as well as at distal sites (2). However, sentinel lymph nodes are also sites where antitumor immune responses can be generated, and thus lymphatic drainage from the tumor likely plays multiple and complex roles in tumor progression. To date, however, there is little understanding of how this communication pathway between tumors and sentinel lymph nodes contributes to the host immune response to the tumor and its progression.

Lymphatic vessels carry fluid and immune cells from peripheral tissues to draining lymph nodes (dLNs), where both components help shape immunity and maintain tolerance to self-antigens (3-5). Without local lymphatic vessels and their associated drainage, peripherally activated dendritic cells (DCs) cannot traffic to the dLNs to activate immune responses (6), and $\mathrm{LN}$ resident immature DCs are not exposed to lymph-borne self-antigens released from

Authorship note: A.W. Lund and M. Wagner contributed equally to this work. Conflict of interest: The authors have declared that no conflict of interest exists. Submitted: October 14, 2014; Accepted: July 7, 2016.

Reference information: / Clin Invest. 2016;126(9):3389-3402. doi:10.1172/JCI79434. extracellular proteases and apoptotic cells for tolerogenic presentation to autoreactive T cells $(7,8)$. Additionally, lymphatic endothelial cells (LECs) themselves can contribute to regional immunity in other ways, including active regulation of fluid drainage (9), direct modulation of DC trafficking and activation $(10,11)$, cellular egress leading to immune resolution $(12,13)$, and direct suppression of lymphocyte activation through steady-state presentation of endogenous self antigens (14) or cross-presentation of draining exogenous antigens $(7,15,16)$. Importantly, lymphangiogenesis is seen in a host of inflammatory situations, including melanoma and other cancers $(1,17)$. However, the role of lymphangiogenesis in inflammation and immunity remains unclear. The abundant clinical and experimental evidence correlating lymphangiogenesis with tumor progression contrasts with reports that lymphangiogenesis in tissue transplantation can promote graft rejection (18), and further confounding the issue are reports suggesting that lymphangiogenesis promotes immune resolution in chronic inflammation $(12,13)$. Therefore, it is likely that lymphatic vessels may serve multiple and complex roles in both the induction and resolution of local immune responses in acute versus chronic inflammation (19).

Inflammation and immunity can play important roles in the initiation, promotion, and metastatic progression of many types of solid tumors. Tumors establish mechanisms to counteract host immunity, and it is the balance between pro- and antitumor inflammatory mediators that likely dictates tumor progression $(20,21)$. While different types of inflammation can either pro- 
A

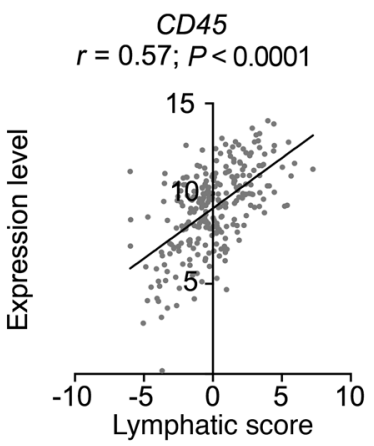

$C D 3 D$

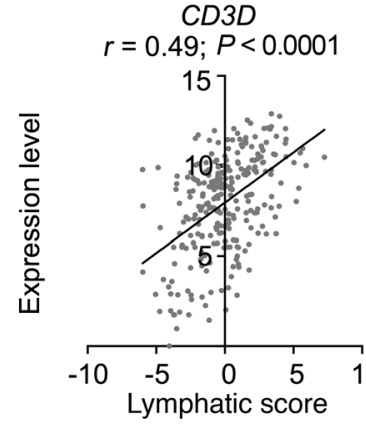

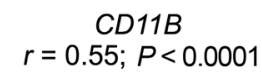
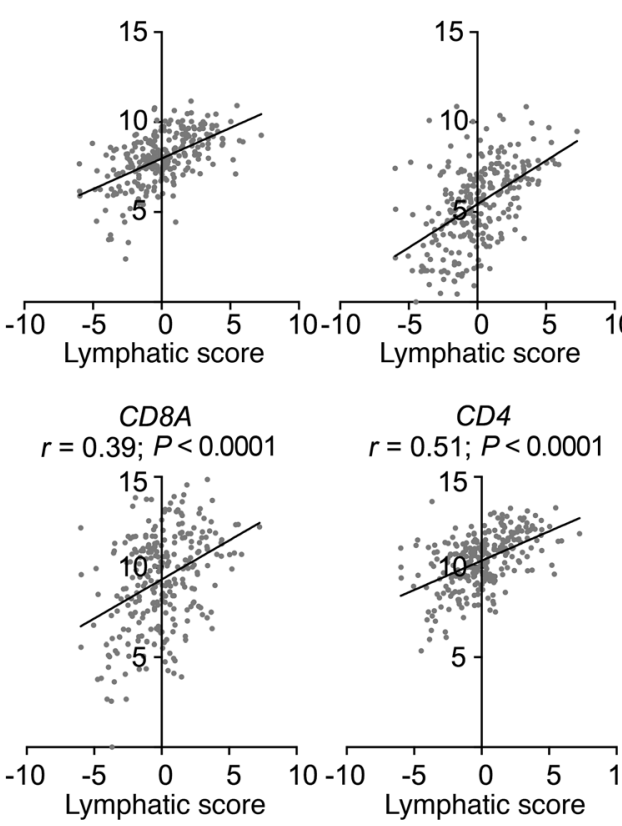

$F 480$
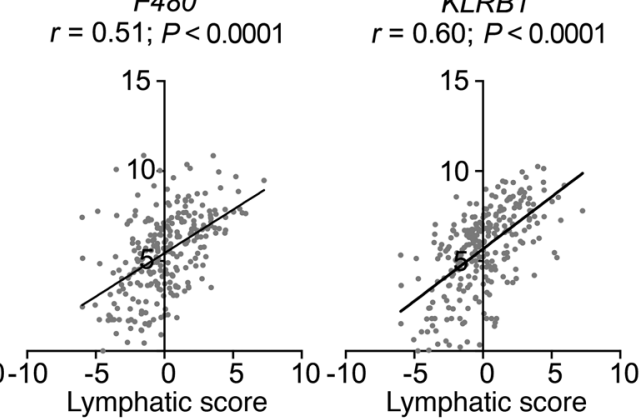

$r=0.60 ; P<0.0001$

Lymphatic score

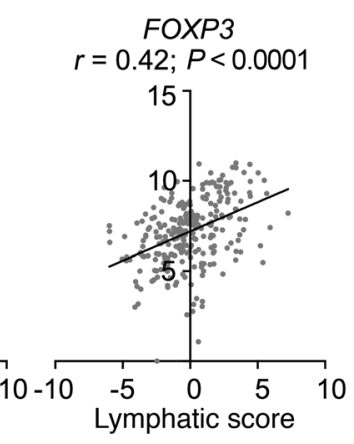

B

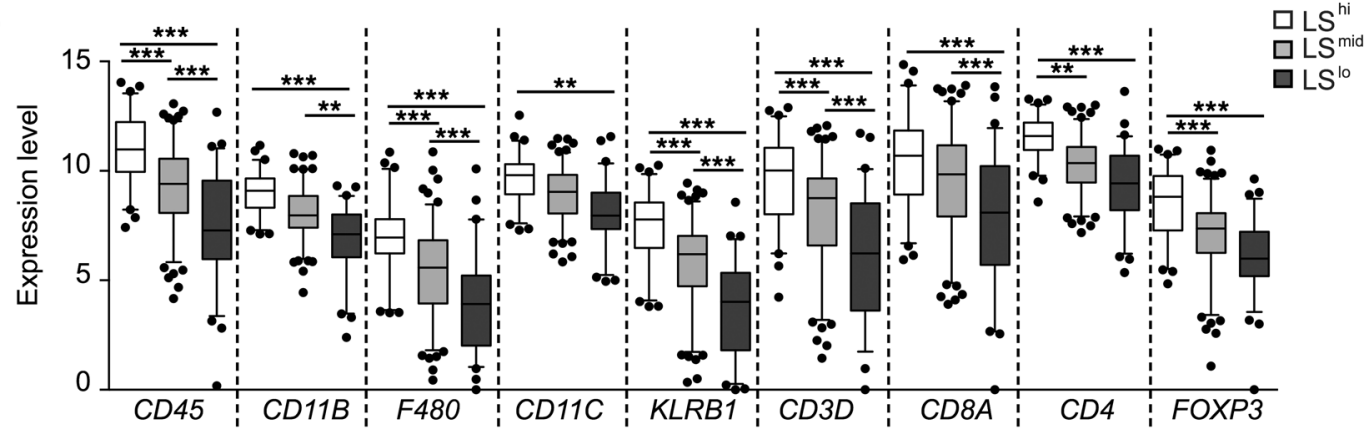

Figure 1. Lymphatic gene expression correlates with immune cell infiltrate in human metastatic cutaneous melanoma. A lymphatic score (LS) was generated using 266 metastatic cutaneous melanoma samples from the Broad Institute's TCGA database. LS was calculated based on relative expression levels of PDPN, LYVE1, and VEGFC in each sample. (A) Plots showing correlative expression of immune cell markers (CD45, CD11B, F480, KLRB1, CD3D, CD8A, CD4, FOXP3) with LS. Pearson's correlation coefficient $(r)$ is shown. (B) Samples were segregated into 3 groups; low (LS' $;<$ [mean - 2/3 SD]), intermediate (LSmid; [mean - 2/3 SD] < LSmid < [mean + 2/3 SD]) and high (LShi; $>$ [mean + 2/3 SD]). Box plots are 5-95 percentile with outliers. One-way ANOVA followed by Tukey's multiple comparisons test was used to determine significance on $n=266$. ${ }^{* *} P<0.01,{ }^{* * *} P<0.005$.

mote or suppress tumor progression in different cancers (21), successful immunotherapy directs productive immune-mediated tumor killing and regression (21-23). Current clinical trials testing checkpoint blockade strategies (e.g., anti-CTLA-4 and anti-PD-1) in metastatic melanoma are demonstrating improved survival in a subset of patients $(24,25)$. Interestingly, those patients that respond to such strategies appear to stratify by the preexistence of immune cell infiltration (26-28), particularly $\mathrm{CD} 8^{+} \mathrm{T}$ cells. The finding that some patients lack tumorinfiltrating lymphocytes and consequently demonstrate poor response to immunotherapy $(28,29)$ indicates that endogenous mechanisms regulating immune induction in the tumor may be responsible for therapeutic resistance (4). While lymphatic vessels and their associated drainage function facilitates communication between tumors and the adaptive immune response, very little is known about how their remodeling may influence the subsequent antitumor immune response. Rather than passive conduits for tumor metastasis, lymphatic vessels are likely context-dependent regulators of local inflammation and immunity.

We recently reported that in a model of murine melanoma, lymphangiogenesis resulted in impaired adaptive immune responses through direct suppression of $\mathrm{CD} 8^{+} \mathrm{T}$ cells (16). On the other hand, as stated above, lymphatic vessels communicate information and transport immune cells, antigens, and signals from the periphery to the $\mathrm{dLN}$, implying that lymphatic vessels must be required for initiating an immune response against a growing tumor. In this work, we explore the relationship between local lymphatic vessels and immune infiltration in melanoma. In human metastatic melanoma, we found strong correlations between the expression levels of lymphatic genes (PDPN, LYVE1, and VEGFC) and inflammatory cell markers and cytokines. In murine melanoma, we asked whether tumors developing in the absence of local 
A

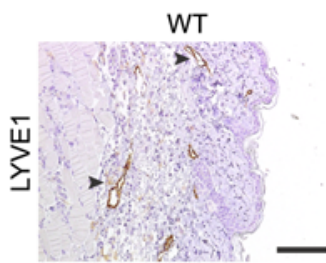

C
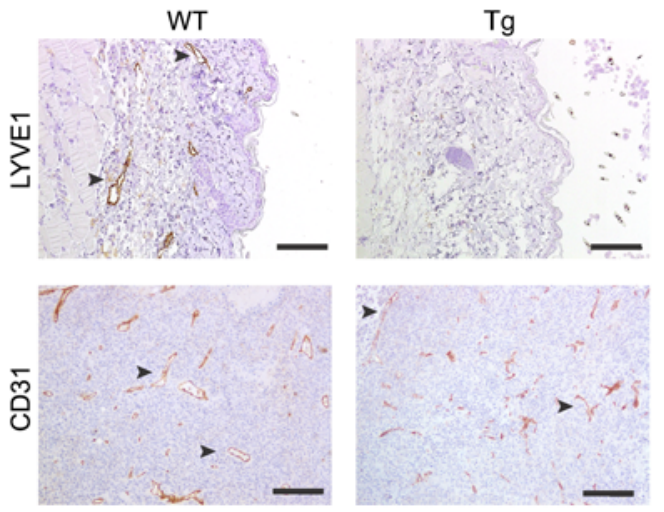

E

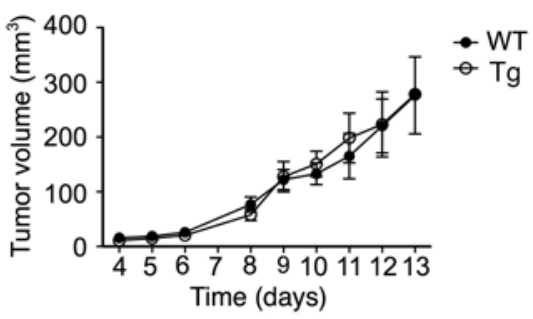

G

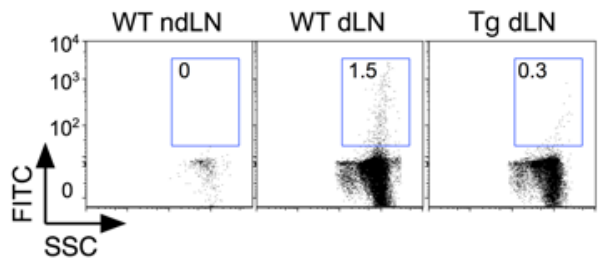

I

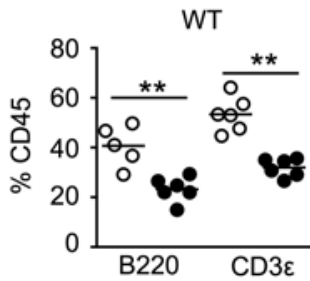

B

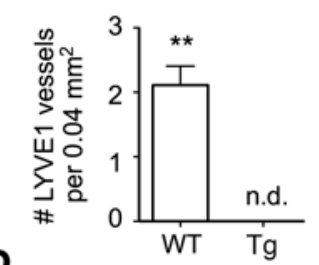

D

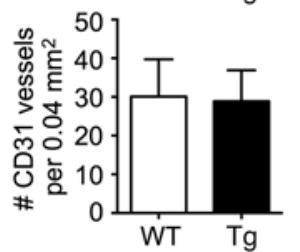

$\mathbf{F}$
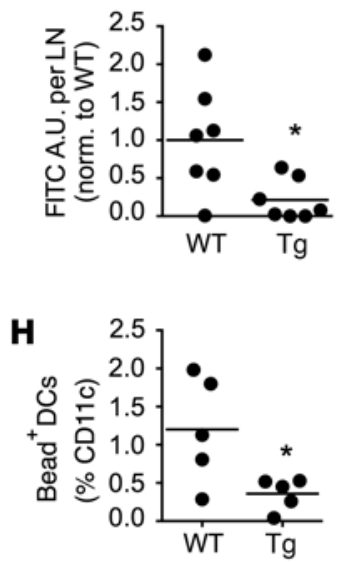

Figure 2. Impaired tumor drainage and DC trafficking to local lymph nodes in K14-VEGFR3-Ig mice. B16F10 tumors were implanted intradermally into WT or K14VEGFR3-Ig transgenic (Tg) mice and excised at day 9. (A) Peritumoral area from WT or Tg mice stained for lymphatic endothelial cells (LYVE1; $n=3$; n.d., not detected) (arrowhead). Scale bar: $50 \mu \mathrm{m}$. (B) Lymphatic vessel density quantified as LYVE1+ structures per $0.04 \mathrm{~mm}^{2}$. Data were compared using Student's unpaired $t$ test. ${ }^{*} P<0.01$ (C) Intratumoral area stained for blood endothelial cells (CD31, $n=3$ ) (arrowhead). Scale bar: $50 \mu \mathrm{m}$. (D) Blood vessel density quantification as $\mathrm{CD} 31^{+}$structures per $0.04 \mathrm{~mm}^{2}$. (E) Tumor growth profiles in WT and Tg mice over 13 days. (F) Fluid drainage from the tumor to the draining lymph node (dLN, brachial) assessed 30 minutes after intratumoral injection of 70-kDa FITC-dextran and plotted as fluorescence intensity in arbitrary units (A.U.) per LN normalized to WT $(n=7)$. (G) DC trafficking from the tumor to the dLN, determined by the quantity of CD11 ${ }^{+} \mathrm{MHCII}^{+} \mathrm{FITC}^{+}$cells in the dLN 24 hours after intratumoral injection of $0.5-\mu \mathrm{m}$ FITC-labeled latex beads and representative flow cytometry dot plots $(n=5)$. (H) Quantification of bead ${ }^{+}$DCs in the LN. (I) Comparison of relative $B$ cell $\left(\mathrm{B}^{2} 2 \mathrm{O}^{+}\right)$and $\mathrm{T}$ cell $\left(\mathrm{CD} 3 \varepsilon^{+}\right)$populations in the dLNs to nondraining lymph nodes ( $n d L N)(n \geq 4)$. Data are represented as the mean \pm SEM. Statistical analysis with Mann-Whitney $U$ test. ${ }^{*} P<0.05,{ }^{* *} P<0.01$. lymphatic vessels, and therefore without communication with the $\mathrm{dLN}$, can acquire an inflammatory microenvironment that is typically seen otherwise. We found that in mice lacking dermal lymphatic capillaries, intradermally implanted B16 melanomas were substantially less infiltrated by immune cells, contained markedly reduced levels of inflammatory cytokines, and failed to induce cellular adaptive immune responses. Given the importance of endogenous immune recognition for successful immunotherapy, lymphatic vessels may provide an intriguing control point for regulating antitumor immunity and response to therapy.

\section{Results}

In human metastatic cutaneous melanoma, lymphatic gene expression correlates with immune parameters. Lymphatic vessel density is associated with melanoma stage and locoregional metastasis (1), but it is unknown how it correlates with tumor immune infiltration. First, we categorized 266 human metastatic cutaneous melanoma samples (The Cancer Genome Atlas, TCGA) (30) to determine whether correlations could be found between the expression levels of lymphatic genes with genes associated with immune infiltration. Of note, in the tumor microenvironment, none of the most common LEC markers - podoplanin (PDPN), LYVE1, and PROX1 - are completely specific to LECs; for example, some tumor-associated macrophages can express LYVE1 (31), and stromal fibroblasts may express PDPN (32). Furthermore, we found that human metastatic melanoma can substantially express PROX1 (Supplemental Figure 1A; supplemental material available online with this article; doi:10.1172/JCI79434DS1). Therefore, we first examined which of these LEC genes correlated most strongly with the lymphatic growth factor VEGFC. Interestingly, we found strong correlations among the gene expression levels of PDPN, $L Y V E 1$, and $V E G F C$, but no correlation between any of these and PROX1, VEGFD, VEGFA, or VEGFB (Supplemental Figure 1B). We therefore defined a lymphatic score (LS) to represent the relative expression levels of PDPN, LYVE1, and VEGFC in each sample (see Methods) and further segregated samples into 3 groups representing low $\left(\mathrm{LS}^{\mathrm{lo}}\right)$, intermediate $\left(\mathrm{LS}^{\mathrm{mid}}\right)$, and high $\left(\mathrm{L} \mathrm{S}^{\mathrm{hi}}\right)$ degrees of lymphatic vessel involvement such that $50 \%$ of the patients were 
A

CD11b

$F 4 / 80$

MHCII

$\mathrm{CD} 3 \varepsilon$
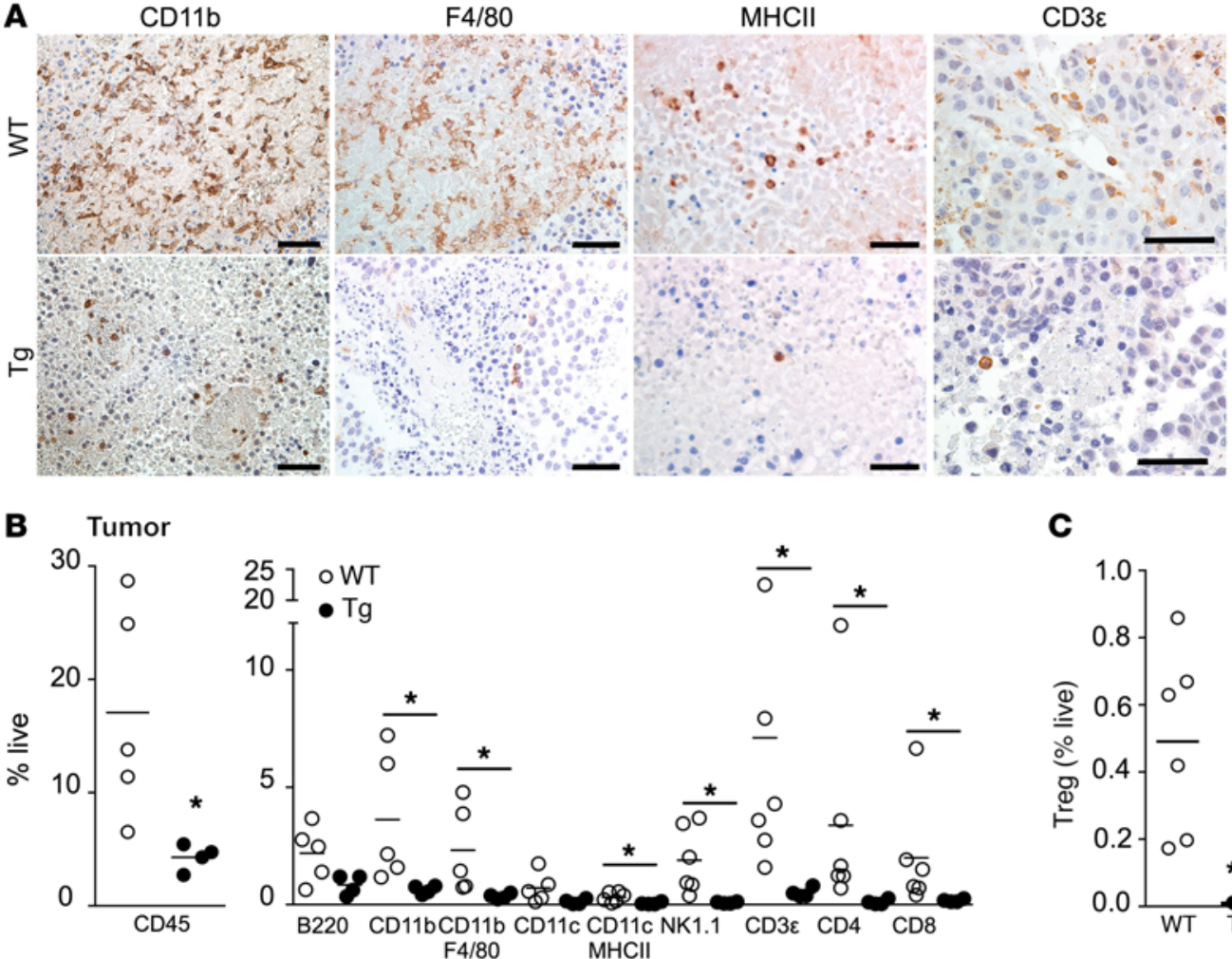

C
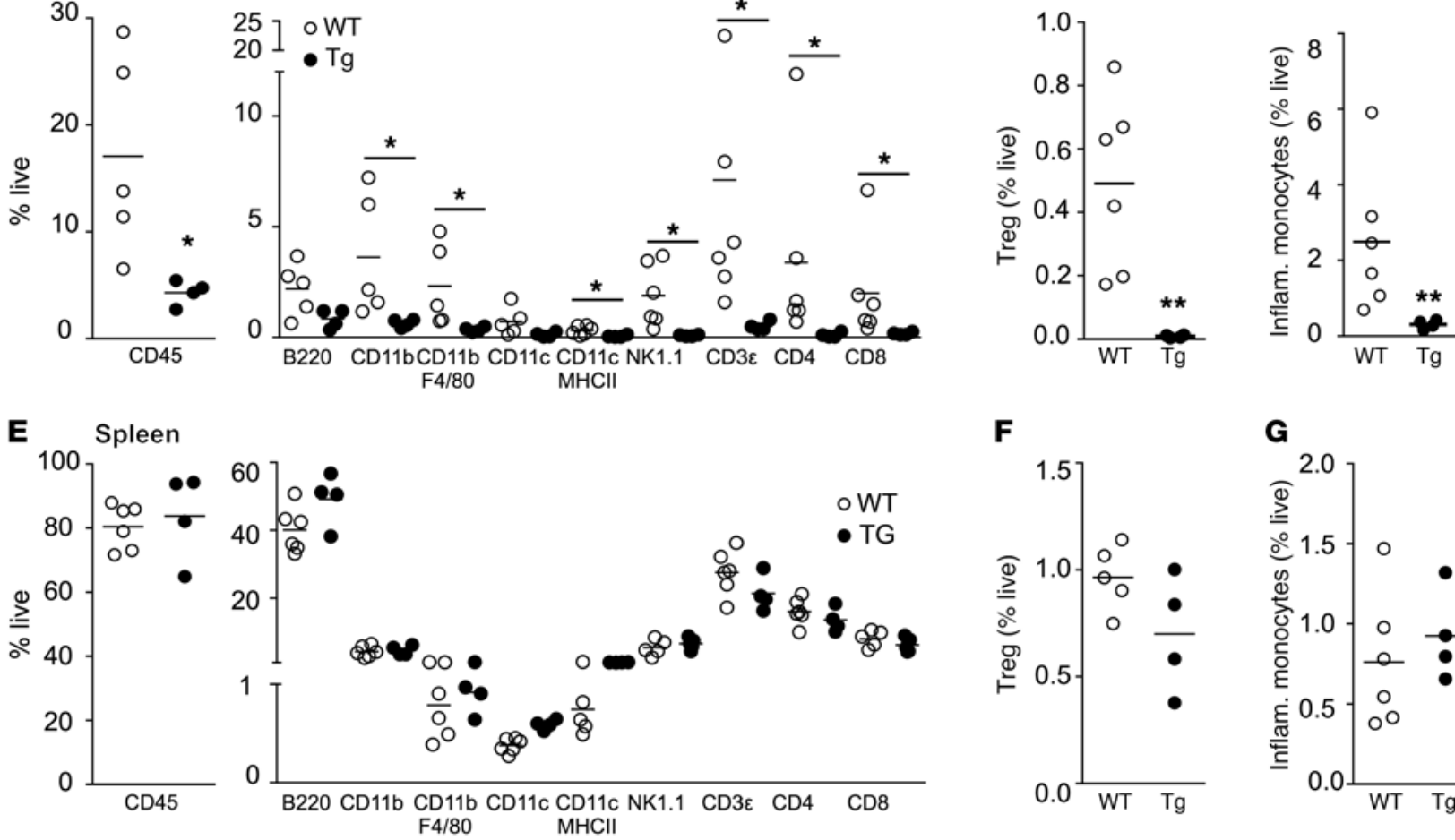

$\mathbf{F}$
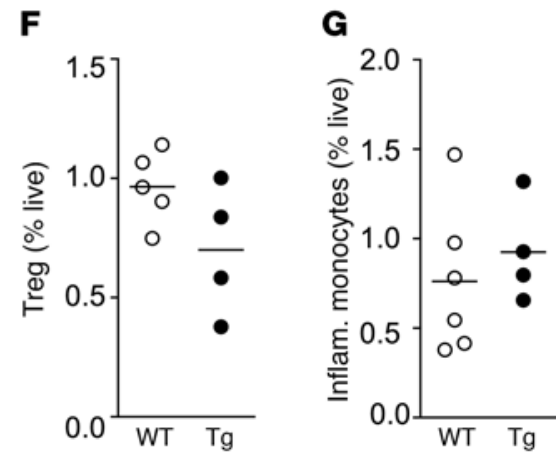

Figure 3. B16F10 melanomas implanted in K14-VEGFR3-Ig mice lack a local inflammatory infiltrate. The inflammatory infiltrate was determined on day 9 in B16F10 tumors grown in WT and K14-VEGFR3-Ig (Tg) mice. (A) Immunohistochemical analysis of tumor immune infiltrates in WT and Tg mice. Scale bars: $50 \mu \mathrm{m}$ (CD11b, F4/80, and CD3E) and $100 \mu \mathrm{m}$ (MHCII). (B-C) Analysis of immune cell populations in the tumor (B-D) and spleen (E-C) by flow cytome-

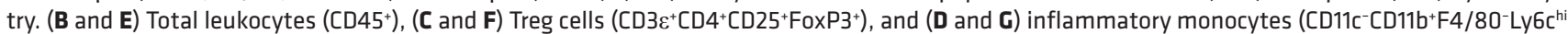
Ly6g $\left.^{-}, n \geq 4\right)$. Statistical analysis with Man-Whitney $U$ test. ${ }^{*} P<0.05,{ }^{* *} P<0.01$

$\mathrm{LS}^{\text {mid }}$ (within $2 / 3$ of the SD around the mean) and $25 \%$ were above and below (Supplemental Figure 2A). This cumulative score correlated very strongly with $V E G F C$, PDPN, and $L Y V E 1$, but again not with $V E G A, V E G F B$, or $V E G F D$ (Supplemental Figure 2B).

Using this data set, we correlated LS with immune markers to evaluate the relationship between lymphatic involvement and immune infiltration. We observed strong positive correlations between LS and a variety of immune cell-specific genes including CD45, CD11B, F480, KLRB1, CD3D, CD8A, CD4, and FOXP3 (Figure $1 \mathrm{~A})$. Furthermore, $\mathrm{LS}^{\text {hi }}$ patients consistently exhibited higher expression of these immune markers than LS $^{\text {mid }}$ and LS ${ }^{\text {lo }}$ (Figure $1 B$ ), indicating strong correlations between lymphatic vessel genes and immune infiltrates in human metastatic cutaneous melanoma.
Dermal lymphatic vessels are required for $L N$ responses to intradermally implanted murine melanoma. Given the positive correlation between LS and immune cell markers in human metastatic melanoma, we wanted to experimentally probe the role of lymphatic vessels in the generation of inflammatory and immune responses against melanoma. We implanted B16F10 melanomas into the dermis of transgenic K14-VEGFR3-Ig mice (referred to in the figures as Tg mice); these mice lack dermal lymphatic vessels due to the expression of a soluble VEGFR3-Ig under the control of the epithelial keratin 14 (K14) promoter, which effectively 'traps' VEGFR3 ligands (VEGF-C and VEGF-D) to prevent local VEGFR3 signaling (33). Histological analysis of tumor tissue demonstrated maintenance of the lymphatic-free phenotype in melanomas in 

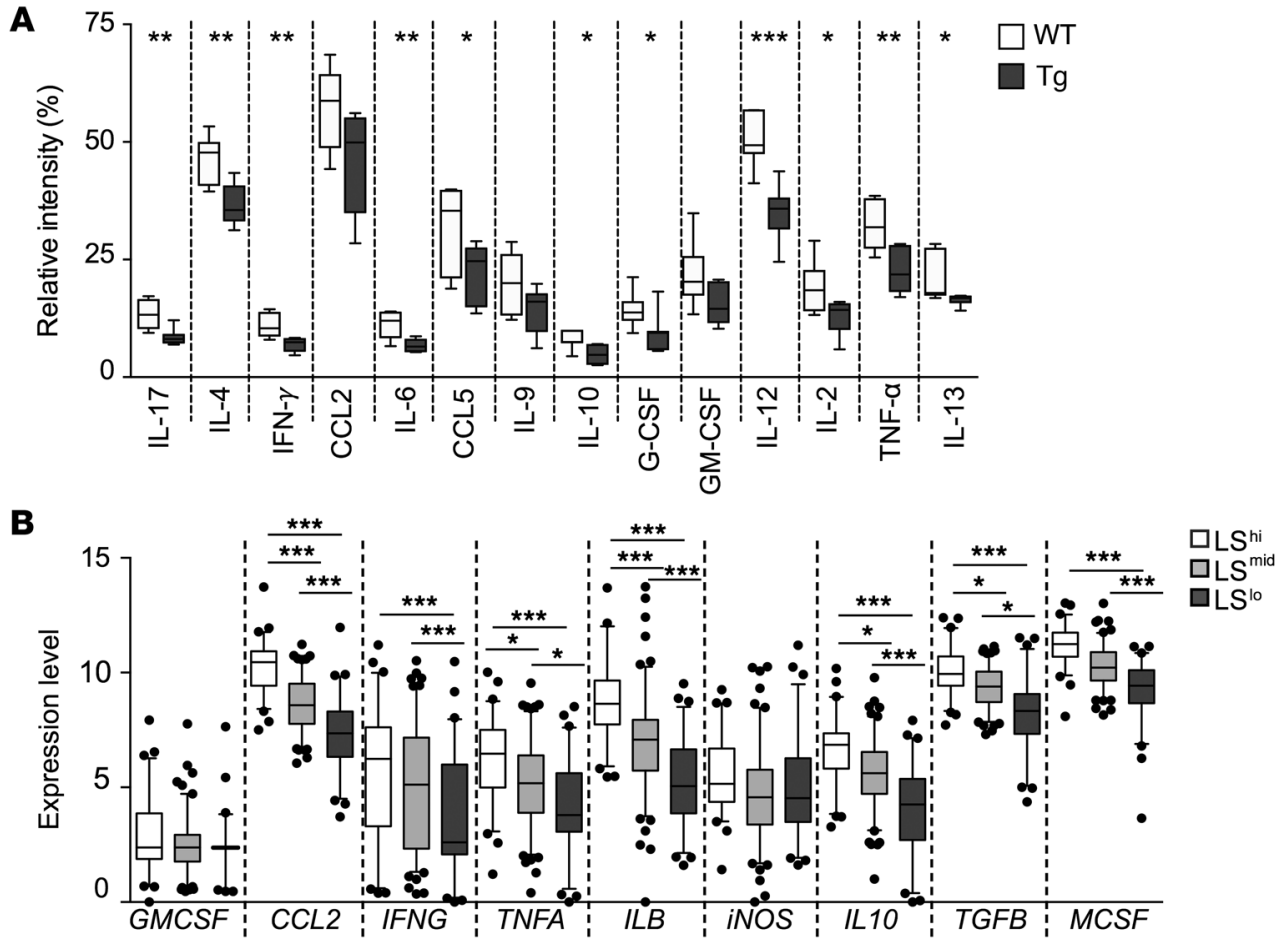

Figure 4. Tumor-associated lymphatic vessels correlate with expression of local inflammatory mediators. (A) The expression of common inflammatory mediators in tumor microenvironments was assessed by cytokine array on tumor tissue lysates from B16F10 melanomas grown in WT or K14-VEGFR3-Ig $(\mathrm{Tg})$ mice $(n=7)$. Relative intensity as compared with internal biotin-conjugated IgG control. Results were compared using Student's unpaired $t$ tests. ${ }^{*} P<0.05$, ${ }^{* *} P<0.01$, ${ }^{* * *} P<0.001$. (B) In human metastatic cutaneous melanoma, lymphatic score (LS) correlated with gene expression of inflammatory cytokines. Box plots generated by segregating samples into 3 groups: $L S^{10}, L^{\text {mid }}$, and $\mathrm{LS}^{\text {hi }}$. Box plots show data within 5-95 percentile with outliers shown as dots. One-way ANOVA followed by Tukey's multiple comparisons test was used to determine significance on 266 samples. ${ }^{*} P<0.05,{ }^{* * *} P<0.005$
K14-VEGFR3-Ig mice (Figure 2, A and B). No significant changes were observed in blood vessel densities as analyzed by numbers of $\mathrm{CD}_{3} 1^{+}$structures per area (Figure 2, C and D); however, the percentage area stained by CD31 was decreased (Supplemental Figure 3), perhaps reflecting smaller vessels as previously reported (34). The lack of dermal lymphatic vessels did not affect tumor growth rates (Figure 2E), and did not appear to affect local collagen density (by picrosirius red staining) or fibroblast phenotype (by $\alpha$-smooth muscle actin ( $\alpha$-SMA) immunohistochemistry) in either normal skin or tumor stroma (Supplemental Figure 4).

To determine the degree of disrupted communication between the tumor and dLN due to missing dermal lymphatic vessels, we quantified the total amount of fluorescently labeled dextran $(70 \mathrm{kDa})$ in the dLNs (brachial) 30 minutes after injection into tumors at day 9 . Not unsurprisingly, we found a roughly 5 -fold decrease in the amount of dextran contained in the dLNs of K14-VEGFR3-Ig mice compared with WT mice (Figure 2F). The axillary LNs of WT mice also contained dextran but to a much lesser extent compared with the brachial, and we found no evidence of dextran in any other LNs including the inguinal (data not shown). We also quantified DC trafficking following intratumoral injection of FITC-labeled latex beads $(0.5 \mu \mathrm{m})$, which are too large to flow into lymphatic vessels and instead must be taken up by DCs in the tumor before being transported to the dLN (35). As expected, DC trafficking was drastically reduced in K14-VEGFR3-Ig mice (Figure 2, G and H), whose LNs had demonstrated lower total cellularity, but a significantly higher percentage of $\mathrm{CD} 3 \varepsilon^{+} \mathrm{T}$ cells before tumor implantation than those in WT mice (Supplemental Figure 5 and Figure 2I).

In WT mice, LNs draining implanted B16 melanomas were enlarged compared with nondraining LNs, with increased fractions of $\mathrm{T}$ cells, reflecting $\mathrm{T}$ cell expansion (Figure 2I). Given the importance of lymphatic transport of fluid, antigens, and DCs in mounting such immune responses, we predicted that these LN changes would be absent in tumor-bearing K14-VEGFR3-Ig mice. Indeed, we found that relative lymphocyte fractions were similar in tumor-draining versus nondraining LNs in transgenic mice (Figure 2I), suggesting a lack of immune response to the growing tumor.

Reduction of tumor-infiltrating leukocytes and inflammatory cytokines in mice lacking dermal lymphatic vessels. Next, we analyzed immune cell infiltration in B16F10 tumors implanted in K14-VEGFR3-Ig and WT mice. Immunohistochemical staining for $\mathrm{CD} 11 \mathrm{~b}, \mathrm{~F} 4 / 80, \mathrm{MHCII}$, and $\mathrm{CD} 3 \varepsilon$ revealed reduced numbers of intratumoral leukocytes (Figure 3A). Flow cytometric analysis of B16F10 tumors demonstrated a 3-fold reduction of CD $45^{+}$cells in tumors growing in K14-VEGFR3-Ig compared with WT mice (Figure 3B). Further breakdown of the $\mathrm{CD} 45^{+}$population demonstrated a broad reduction of the most dominant immune subsets, including B cells $\left(\mathrm{B} 22 \mathrm{O}^{+}\right)$, antigen-presenting cells $\left(\mathrm{CD} 11 \mathrm{~b}^{+}\right.$ and $\mathrm{CD} 11 \mathrm{c}^{+}$) and $\mathrm{CD} 3 \varepsilon^{+} \mathrm{T}$ cells as well as Treg cells $\left(\mathrm{CD} 3 \varepsilon^{+}\right.$ $\mathrm{CD}^{+}{ }^{+} \mathrm{CD} 25^{+} \mathrm{FoxP}^{+}$) and immature inflammatory monocytes

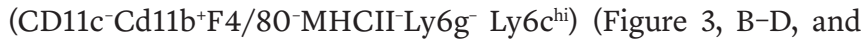
Supplemental Figure 6). These differences were due to immune infiltration into the tumor, since no differences were observed in homeostatic cutaneous and splenic immune populations (Supplemental Figure 7). Similarly, no differences were found between the systemic leukocyte populations (spleen) in tumor-bearing WT and K14-VEGFR3-Ig mice (Figure 3, E-G).

Mirroring the decrease in immune cell infiltrates, we found substantial decreases in the most abundant inflammatory cytokines in tumors growing without lymphatic capillaries (Figure 4A and Supplemental Figure 8, A-C). Similarly, in human cutaneous metastatic melanoma, gene expression of inflammatory cytokines correlated with LS (Supplemental Figure 8D). Again, stratification of patients 
A

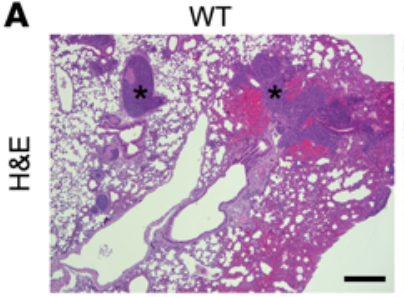

C

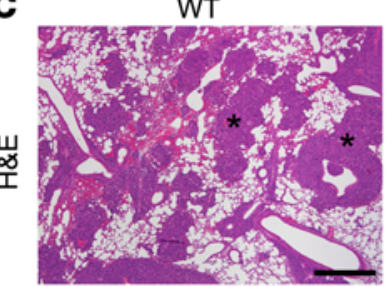

$\mathbf{F}$

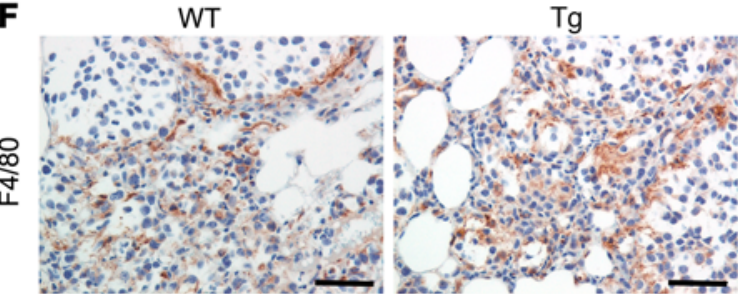

$\mathrm{Tg}$

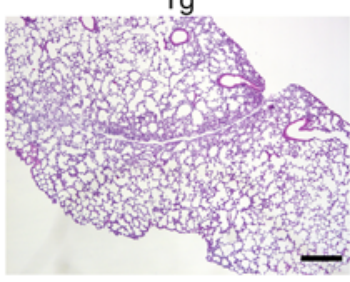

$\mathrm{Tg}$

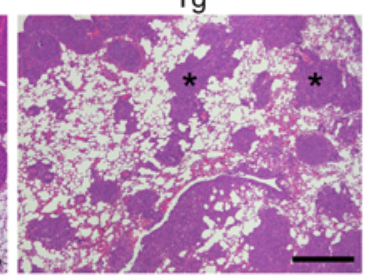

$\mathrm{Tg}$

G

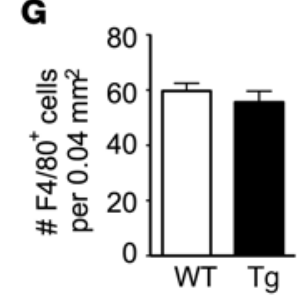

E

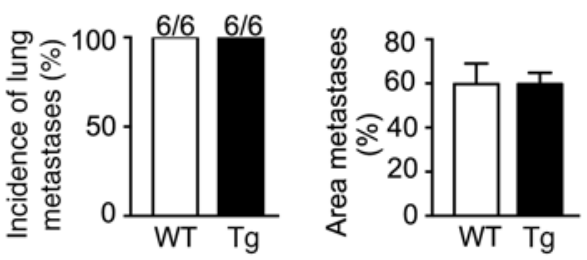

Figure 5. Spontaneous lung metastasis from primary B16F10 melanomas is decreased in K14-VEGFR3-Ig mice. (A) Histological determination of spontaneous lung metastases from orthotopically implanted B16F10 tumors. Scale bars: $1 \mathrm{~mm}$. Paraformaldehyde-fixed, paraffin-embedded sections were stained with hematoxylin and eosin (H\&E). (B) Quantification of incidence from 15 WT and 16 K14-VEGFR3-lg (Tg) mice. Statistical analysis with Fisher exact test. ${ }^{* *} P<0.01$. (C) Histological determination of metastatic colonization in the lung after intravenous injection shows no differences between WT and Tg mice. Scale bars: $200 \mu \mathrm{m}$. $\left(^{*}\right)$ marks metastatic nodules. (D) Quantification of metastatic incidence and (E) area fraction of lung bearing metastatic nodules $(n=6)$. Data represented as the mean \pm SEM. (F) Analysis of macrophage infiltrate in lung metastases 14 days following intravenous injection by immunohistochemistry on paraformaldehyde-fixed paraffin-embedded tissues (F4/80). Scale bars: $50 \mu \mathrm{m}$. (G) Density of F4/80+ macrophages (number per $0.04 \mathrm{~mm}^{2}$ ) analyzed from immunostained sections $(n=6)$. Data represented as the mean \pm SEM.

into $\mathrm{LS}^{\mathrm{hi}}, \mathrm{LS}^{\mathrm{mid}}$, and $\mathrm{LS}{ }^{\mathrm{lo}}$ populations predicted high, intermediate, and low expression of each inflammatory factor, respectively (Figure 4B), suggesting that LS strongly correlates with leukocyte infiltration and cytokine expression in human melanoma.

We confirmed this phenotype in a second model of lymphatic vessel dysfunction (Chy mice, C3H background). Chy mice harbor a heterozygous inactivating mutation in the tyrosine kinase domain of VEGFR3 (36), causing a decrease in lymphatic transport from skin (37). Notably, in this model, dermal lymphatic vessels have been shown to be mostly absent in extremities (i.e., forelimb and ear skin), while trunk skin contains sparse residual lymphatic capillaries able to support cellular egress from tissue (6). We therefore implanted syngeneic C3HBA breast carcinoma cells into ear dermis of Chy mice and WT littermates. Tumors demonstrated enhanced growth in Chy mice (data not shown) and surprisingly, demonstrated some LYVE1 $1^{+}$structures surrounding tumors, although at a significantly lower density than tumors in WT littermates (Supplemental Figure 9). This decrease in lymphatic vessels corresponded to decreased $\mathrm{CD}_{4} 5^{+}$leukocytic infiltration and a notable lack of $\mathrm{CD} 3 \varepsilon^{+} \mathrm{T}$ cells in and around the tumor (Supplemental Figure 9). Although the differences in leukocytic infiltration in tumors growing in Chy mice were not as pronounced as those in tumors in K14-VEGFR3-Ig mice, this result is consistent with the decrease in lymphatic density being less pronounced as well.
Given the presence of soluble VEGFR3 within the adult K14VEGFR3-Ig mice, we sought to verify that our results were due to absence of a lymphatic vasculature rather than inhibition of local VEGFR3 signaling during tumor development. To test this, we implanted tumors into WT C57BL/6 mice and administered a VEGFR3-blocking antibody at the time of tumor implantation and every 3 days thereafter (Supplemental Figure 10A). The inhibition of VEGFR3 signaling during tumor growth did not significantly alter fluid drainage out of tumors to dLNs (Supplemental Figure 10B), indicating that preexisting lymphatic vessels were functional in maintaining communication with the dLN. Additionally, no differences were observed within leukocyte populations in either the tumors (Supplemental Figure 10, C and D) or spleens (Supplemental Figure 10, E and F).

Decreased metastatic dissemination in K14-VEGFR3-Ig mice. Following sacrifice at later time points, we quantified the incidence of spontaneous lung metastases. In WT mice, 10 of 16 implanted tumors metastasized and colonized lungs, while only 2 of 15 tumors implanted in K14-VEGFR3-Ig mice successfully colonized the lung (Figure 5, A and B). To rule out the possibility that circulating tumor cells in transgenic mice had decreased potential to extravasate into and colonize lung tissue, we injected tumor cells intravenously and found no differences in either incidence of lung colonization or percentage of lung area covered by metastatic tumor cells between WT and transgenic mice (Figure 5, C-E). Considering that the lym- 
A

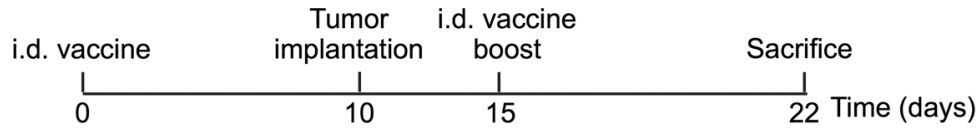

B
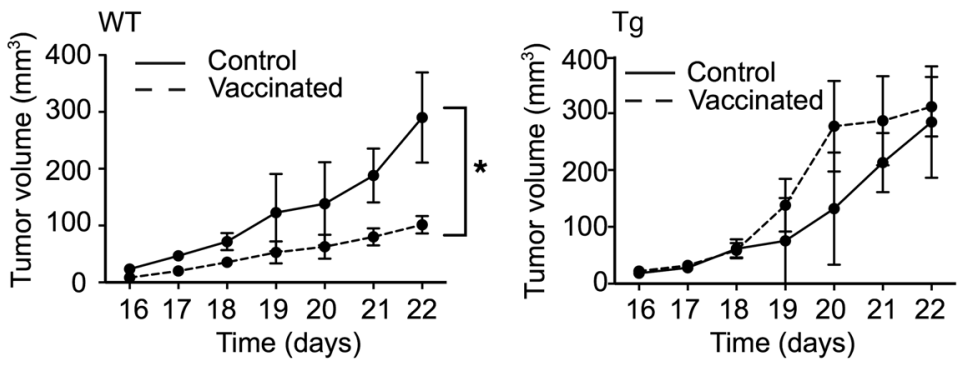

C

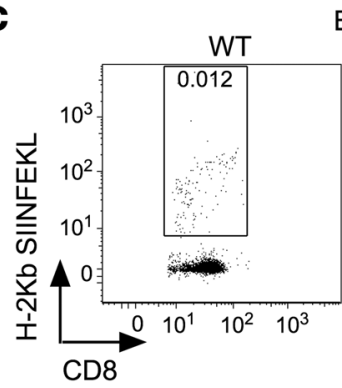

Blood

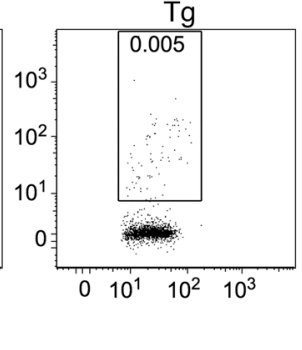

D

$\mathbf{E}$

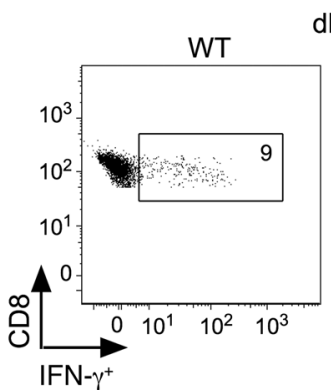

$\mathrm{dLN}$

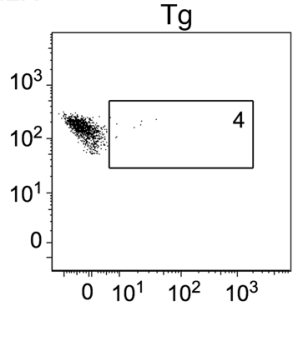

G

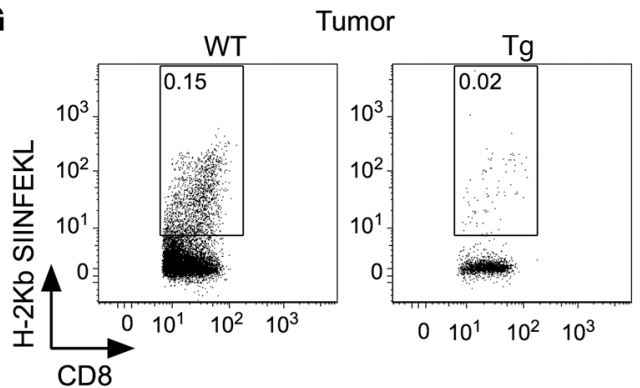

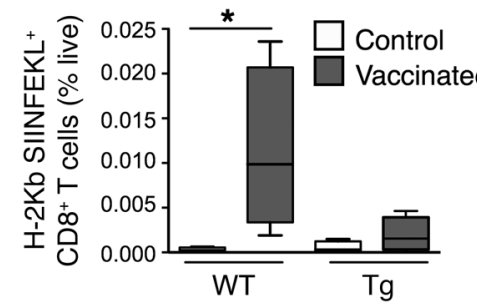

$\mathbf{F}$

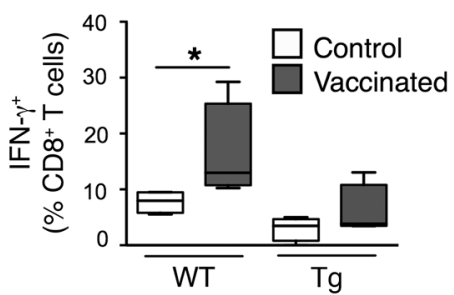

H

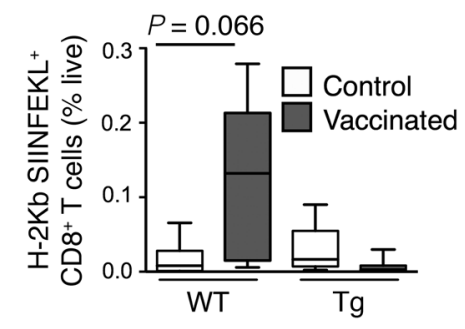

Figure 6. K14-VEGFR3-Ig mice demonstrate impaired antitumor immunity in response to dermal vaccine delivery. (A) Experimental schematic. WT or K14-VEGFR3-lg (Tg) mice were vaccinated intradermally (i.d.) in the forelimbs with either 10 $\mu \mathrm{g}$ ovalbumin (OVA) and $10 \mu \mathrm{g}$ LPS, or 10 $\mu \mathrm{g}$ LPS alone. After 10 days, B16F10.0VA tumor cells $\left(0.5 \times 10^{6}\right)$ were injected i.d. in the back skin and the vaccine was boosted, at the same dose, on day 15 . Mice were sacrificed on day 22 (12 days after tumor implantation). (B) Tumor growth was slower in vaccinated vs. control mice for melanomas in WT (left), but not Tg (right) mice. $P$ values determined by regression analysis. ${ }^{*} P<0.05$. (C) Representative plots and (D) quantification of circulating antigen-specific CD8 ${ }^{+} \mathrm{T}$ lymphocytes as detected by $\mathrm{H}$-2Kb SIINFEKL pentamer staining on day 12. (E) Representative flow cytometry plots showing intracellular cytokine staining for IFN- $\gamma$ and (F) quantification of IFN- $\gamma^{+} \mathrm{CD}^{+} \mathrm{T}$ cells following peptide restimulation of tumor-draining LN cells on day 12. (G) Representative flow cytometry plots and $(\mathbf{H})$ quantification of tumor-infiltrating antigen-specific CD8 ${ }^{+} \mathrm{T}$ cells detected by $\mathrm{H}-2 \mathrm{~Kb}$ SIINFEKL pentamer staining on day $12(n=5$, in at least 2 separate experiments). Box and whisker plots show data from min to max. Statistical analysis with 1-way ANOVA. ${ }^{*} P<0.05$. phatic vasculature in the lung is intact in K14-VEGFR3-Ig mice, we also analyzed macrophage infiltration into these metastatic lung nodules and found no differences in macrophage infiltration compared with WT mice (Figure 5, F and G).

Dermal vaccination does not control primary tumor growth in the absence of lymphatic vessels. We previously reported evidence that ovalbumin-expressing (OVA-expressing) B16F10 (B16F10.OVA) tumors respond to prophylactic vaccination against OVA (16), where intradermal vaccination resulted in potent activation of immune cells within the $\mathrm{dLN}$, and activated $\mathrm{CD} 8^{+} \mathrm{T}$ cells homed to tumors and controlled primary tumor growth. We applied this model vaccine to our K14-VEGFR3-Ig mice to determine whether immune activation would be delayed in these animals. Ten days before tumor implantation, naive mice were vaccinated against OVA $(10 \mu \mathrm{g})$ with LPS $(10 \mu \mathrm{g})$ intradermally in forelimbs. Subsequently, a vaccine boost was given 5 days after implantation and tumor growth was monitored for 1 week (Figure 6A). Interestingly, while vaccination controlled tumor growth in WT mice, it had no effect on tumors in K14-VEGFR3-Ig mice (Figure 6B). The altered responsiveness to the vaccine correlated with an impaired number of circulating antigen-specific (MHCI-SIINKFEL) $\mathrm{CD}^{+} \mathrm{T}$ cells (Figure 6, C and D), decreased IFN- $\gamma$ production by $\mathrm{CD}^{+} \mathrm{T}$ cells in dLNs following restimulation (Figure 6, E and F), and fewer infiltrating antigen-specific $\mathrm{CD}^{+} \mathrm{T}$ cells in tumors of K14-VEGFR3-Ig 
A

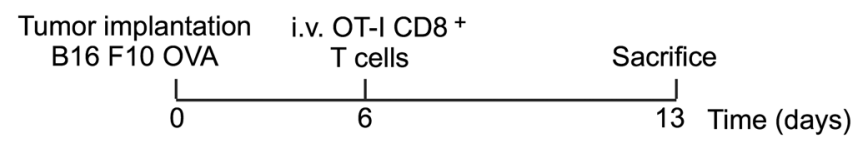

B
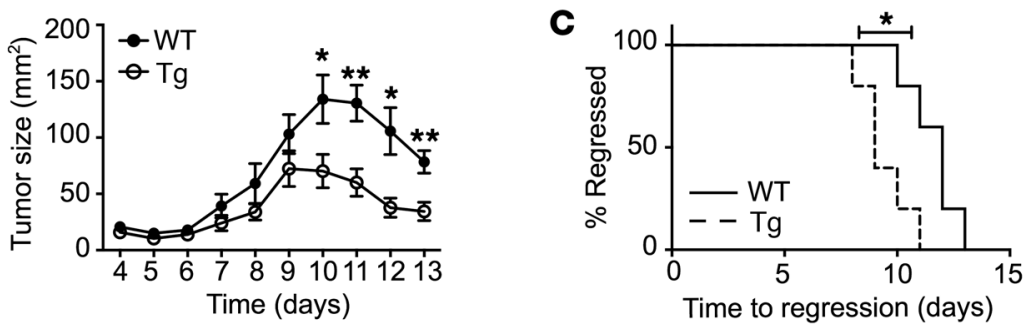

D

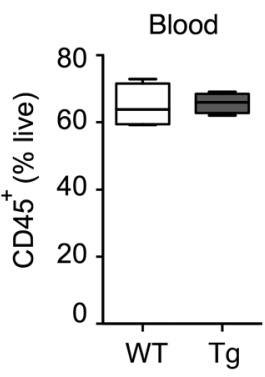

G

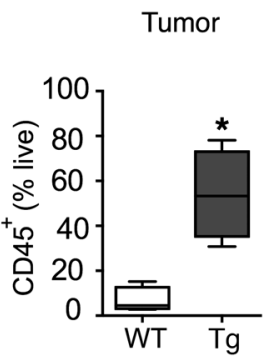

E

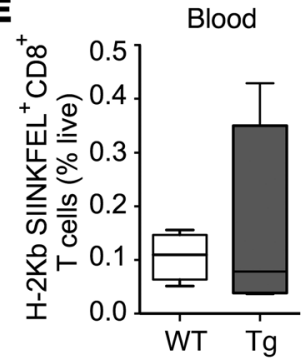

H

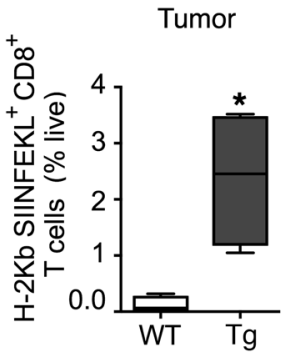

$\mathbf{F}$

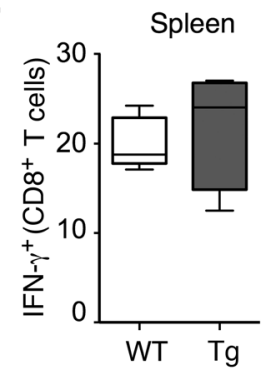

I

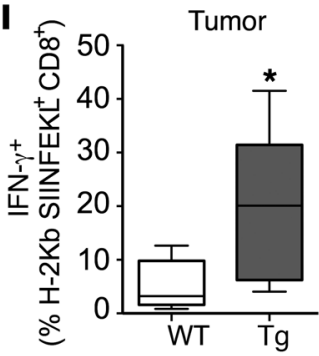

Figure 7. Improved efficacy of adoptively transferred anti-OVA effector $C D 8^{+} T$ cells against OVA-expressing melanomas implanted in K14-VEGFR3-Ig mice.

(A) Experimental schematic. B16F10.0VA tumor cells $\left(0.5 \times 10^{6}\right)$ were injected intradermally (i.d.) into either WT or K14-VEGFR3-Ig (Tg) mice, and after 6 days, activated OT-I CD8 ${ }^{+} \mathrm{T}$ cells were injected intravenously (i.v.). Mice were sacrificed 7 days following transfer. (B) Tumor growth profiles and (C) Kaplan-Meier curve showing relative times to tumor regression $(n=5)$. (D and E) Flow cytometric analysis of circulatory (D) CD45 $5^{+}$cells or (E) OT- 1 cells at day 13 , and (F) IFN- $\gamma^{+}$ $T$ cells in spleen following restimulation. (G-K) Flow cytometric analysis of tumor infiltrating (G) leukocytes (CD45), (H) antigen-specific CD8 ${ }^{+} \mathrm{T}$ cells $(\mathrm{H}-2 \mathrm{~Kb}$ SIINFEKL $L^{+}$, and (I) IFN- $\gamma^{+} \mathrm{CD}^{+} \mathrm{T}$ cells following in vivo brefeldin A treatment. Box and whisker plots show data from min to max. Statistical analysis with Mann Whitney $U$ test. ${ }^{*} P<0.05,{ }^{* *} P<0.01$ in at least 2 separate experiments. mice (Figure 6, G and $\mathrm{H}$ ). These findings are consistent with our previous findings that K14-VEGFR3-Ig mice respond in a delayed manner to vaccination (8).

Tumors established in the absence of lymphatic vessels are more vulnerable to transferred T cells. Although K14-VEGFR3-Ig mice were unable to induce a potent adaptive $\mathrm{T}$ cell response against B16F10.OVA tumors expressing a model antigen, the absence of tumor-infiltrating Treg cells and inflammatory monocytes indicates a more permissive microenvironment for effector $\mathrm{T}$ cell function. We activated antigen-specific $\mathrm{CD}^{+} \mathrm{T}$ cells (OTI) ex vivo with SIINFEKL-loaded splenic DCs and IL-2 (10 U/ $\mathrm{ml}$ ) for 4 days. Five days after implantation, $10^{6}$ activated CD ${ }^{+}$ $\mathrm{T}$ cells were transferred intravenously and tumor growth was monitored for 9 days (Figure 7A). As expected, transferred, activated CD8 ${ }^{+} \mathrm{T}$ cells significantly controlled growth of B16F10. OVA cells in WT mice (Figure 7B). Interestingly, regression of B16F10.OVA growth in K14-VEGFR3-Ig mice was significantly increased when compared with littermates, with respect to both level of regression and time to onset (Figure 7, B and C). Total circulating CD $45^{+}$leukocytes (Figure 7D), antigen-specific T cells (Figure 7E), or functional IFN- $\gamma$ production by $\mathrm{CD}^{+}$ $\mathrm{T}$ cells in spleen following restimulation (Figure $7 \mathrm{~F}$ ) were equal in both strains. Within tumor microenvironments, however, a significant infiltration of $\mathrm{CD} 45^{+}$cells was observed in $\mathrm{K} 14$ -
VEGFR3-Ig mice over controls (Figure 7G). This corresponded with an increase in tumor-infiltrating antigen-specific $\mathrm{CD} 8^{+} \mathrm{T}$ cells (Figure $7 \mathrm{H}$ ). To test $\mathrm{CD} 8^{+} \mathrm{T}$ cell functionality within tumor microenvironments, we performed an in vivo brefeldin A assay for intracellular staining of IFN- $\gamma$. Enhanced IFN- $\gamma$ production was observed in $\mathrm{T}$ cells infiltrating tumors lacking a lymphatic compartment (Figure 7I).

\section{Discussion}

Melanoma is a particularly attractive target for immunotherapy given its inherent immunogenicity and high frequency of infiltrating intratumoral lymphocytes in a major subset of patients (38, 39). Furthermore, several immunotherapeutic strategies to treat metastatic melanoma appear to be dependent upon preexistence of an immune infiltrate $(26,27,40)$. It remains unclear, however, what determines the propensity of an individual patient to generate an endogenous response against their tumor. In this study, we examined the role that lymphatic vasculature plays in generation of endogenous inflammatory and adaptive immune responses within and against a developing malignancy. Using a cohort of 266 cutaneous metastatic melanoma patients, we established an LS based on expression of the lymphatic vessel markers PDPN and $L Y V E 1$. These markers significantly correlated with each other as well as with VEGFC. Interestingly, LS did not correlate with oth- 
er VEGFs, indicating specificity to the VEGF-C/VEGFR3 signaling axis. Patients who scored high for LS consistently had higher expression of immune and inflammatory markers, whereas those who scored low presented with decreased expression. To the best of our knowledge, this study provides the first human data that indicates that expression of lymphatic vessel markers may be predictive of immune microenvironments in metastatic melanoma.

To model the role of lymphatic vessels in the induction of tumor inflammation and immunity, we used K14-VEGFR3-Ig mice in which transgenic expression of the VEGFR3-Ig fusion protein under control of the K14 promoter effectively inhibits dermal lymphatic vessel formation (33). This phenotype persists in skin throughout adulthood, while systemic lymphatic networks, though aberrant early, are restored over time (i.e., heart, diaphragm) (33). A decrease in tumor incidence and delayed onset in response to chemical carcinogenesis was recently reported in that model when compared with controls (34), which was notably in a different background than ours (FVB vs. C57BL/6). Initiated tumors, however, progressed normally in transgenic mice with no difference in tumor growth observed, and decreased rates of initiation correlated with decreased local immune populations in skin of transgenic mice. Importantly, the authors observed decreased homeostatic myeloid populations in skin as well as changes in immune infiltrate in and around developing tumors and suggested that impaired local inflammation contributes to impaired initiation. Here, in the C57BL/6 background, we did not observe differences in steady-state cutaneous immune populations in transgenic mice and suggest that in this background the absence of dermal lymphatic vessels impaired local inflammation in response to tumor implantation. Lymphoid populations were, however, altered at homeostasis, likely due to disrupted immune architecture and reduced cellularity already reported in these mice (8).

Lymphatic vessels and their associated fluid drainage thereby play a critical role in initiating host responses to a developing tumor, and while completely absent in dermis of our experimental model, it is reasonable to imagine that significant remodeling of local lymphatic vessels, which will functionally perturb and alter fluid flow rates, may similarly influence inflammation and host antitumor immunity. Consistent with this, a $60 \%$ reduction of lymphatic vessels in Chy mice resulted in a more modest $40 \%$ reduction in $\mathrm{CD} 45^{+}$infiltrate. The direct mechanism by which lymphatic vessels and their associated transport of fluid and cells impact local inflammation remains an interesting question. The current paradigm suggests that chemokines and cytokines released by tumor cells induce immune infiltration, sequentially leading to production of inflammatory mediators, tumor cell killing, activation of DC homing to LNs, and induction of adaptive immunity. Our data may indicate that in the absence of important feed-forward mechanisms leading to adaptive immunity, the growing tumor fails to facilitate the accumulation of inflammatory mediators over time, and thus the reduced levels noted in our study. These observations are consistent with a role for lymphatic vessels in immune resolution where cell egress mediates resolution of inflammation in skin models (12), but indicates that with limited lymphatic drainage, in the context of a growing tumor, inflammation is not initiated and therefore resolution responses are not required.
While our results demonstrate that induction of local inflammation requires functional lymphatic drainage, systemic leukocyte populations and accumulation of Treg cells and inflammatory monocytes were similar in both strains. Inflammatory monocytes expanded in the spleens of both K14-VEGFR3-Ig and WT mice but did not infiltrate the tumor microenvironment. Consistently, splenic inflammatory monocyte populations have been reported to be a marker of tumor progression, yet are not predictive of infiltration and function within tumor microenvironments $(41,42)$. As such, our data indicate a separation of locoregional inflammation and systemic responses. Our work interestingly may support the concepts that targeting a sentinel LN is a viable option for optimal induction of local immunity (43) and local biomarker validation in tumor microenvironments may provide enhanced value over those found in blood.

In addition to lymphatic vessels, certain types of macrophages can express VEGFR3 and consequently their signaling may be affected in this model. On the other hand, VEGFR3 expression was previously not observed on cutaneous macrophages in either untreated, DMBA-TPA-treated skin, or squamous cell carcinomas (34). We provide specific experimental evidence, however, to demonstrate that active VEGFR3 signaling during tumor development does not mimic the observations found in mice completely lacking dermal lymphatic vessels. Importantly, this experiment also makes the distinction between lymphangiogenic or hyperplastic lymphatic networks and functionality of an existing network. Administration of an anti-VEGFR3 antibody importantly inhibits lymphangiogenic responses and other VEGFR3-mediated biology (macrophage and angiogenesis), but does not affect the existing vasculature. Using this model, we observed no significant change in any cell population, including macrophages, as a result of this VEGFR3 inhibition and furthermore, immunohistochemical analysis of lung nodules following intravenous tumor cell administration revealed equivalent amounts of macrophage infiltration within the same genetic background. Consequently, impairment of immune infiltration was restricted to regions of high transgene expression that lack lymphatic vessels, and was not a result of decreased signaling during tumor development.

Angiogenic blood vessels regulate lymphocyte entry into tissues through upregulation of adhesion molecules in response to local inflammation (44) and may utilize VEGFR3 during angiogenic sprouting (45). This raises the possibility that subtle changes in vascular endothelium may contribute to the observed decrease in immune cell infiltration observed in transgenic mice rather than a direct effect of impaired lymphatic drainage. The density of $\mathrm{CD} 1^{+}$structures in B16F10 tumors was similar in WT and transgenic mice, though we did observe a decrease in the percentage area stained with our CD31 marker. However, it is difficult to separate vasculature changes that may result from soluble VEGFR3 and those that would result from altered local inflammation, which is clearly altered in our model. Conversely, altered tumor vasculature and resulting hypoxia may also influence inflammation and progression, highlighting the complex interactions that are at play within tumor microenvironments. However, in healthy skin, K14-VEGFR3-Ig animals demonstrated robust $\mathrm{T}$ cell infiltration following dermal challenge in a contact hypersensitivity assay (8). Similarly, we observed increased immune cell infiltra- 
tion following intravenous transfer of effector $\mathrm{CD}^{+} \mathrm{T}$ cells, both of total CD $45^{+}$cells and antigen-specific CD ${ }^{+} \mathrm{T}$ cells, indicating that tumor-associated endothelium is permissive of infiltration.

In addition, previous reports have described dermal thickening in transgenic mice (34), which, if fibrotic, may influence immune cell infiltration and function. Other architectural changes within skin present in the K14-VEGFR3-Ig mice may also influence immune infiltration in the tumor context or metastasis. Subcutaneous lipid deposition is not elevated in K14-VEGFR3-Ig mice, unlike the Chy mutant mice (37). Chy mice, but not K14VEGFR3-Ig mice, exhibit increased dermal collagen content, and K14-VEGFR3-Ig mice demonstrate enhanced hydraulic conductivity as compared with WT littermates (37). Consistent with this, no difference between collagen content or $\alpha$-SMA was detected in skin of transgenic mice, either at steady state or in association with an implanted tumor. In the context of both of these tissue microenvironments, however, we observed reduced leukocytic infiltration into intradermal tumors. Interestingly, in Chy mice we only observed a $60 \%$ reduction in lymphatic vessel density in the ear and yet this was sufficient to alter the local infiltrate. These data are consistent with the human data in which the extent of lymphatic vessel gene expression was predictive of local infiltration.

Interestingly, systemic metastasis was reduced in K14-VEGFR3Ig mice compared with WT mice. This was not a result of an impaired microenvironment within the lung itself, as intravenously administered tumor cells were able to effectively colonize lungs in both strains. In our case, LN metastasis may be a route of systemic dissemination, as tumor cells move through collecting lymphatic vessels remaining in subcutaneous tissue (37) and eventually re-enter circulation via the thoracic duct (17). While direct evidence for lymphatic vessel-mediated systemic spread is lacking and the efficacy of sentinel LN resection as a means of preventing metastatic spread is controversial $(46,47)$, these data indicate that lymphatic vessels contribute to a more metastatic phenotype within primary tumor microenvironments. In fact, impaired local inflammation that results from loss of lymphatic vessels may itself alter metastatic phenotype, as immune cells play an important role in directing tumor cell exit via hematogenous vasculature through secretion of matrix metalloproteinases, growth factors, and chemokines (48). In PyMT mice with a recessive null mutation in the $C s f 1$ gene, macrophage recruitment was impaired, leading to delayed progression to malignancy and lung metastasis (49). Furthermore, macrophages are often found tethered to blood vessels where they facilitate the exit of metastatic tumor cells (50). We observed reduction in both inflammatory cells, including macrophages, as well as their soluble mediators, suggesting that mechanisms of metastasis would be impaired in our model. Whether the change in metastatic potential observed in K14-VEGFR3-Ig mice results from the lack of direct lymphatic access to circulation or the altered immune microenvironment within tumors and sentinel LNs remains difficult to uncouple.

In our OVA vaccination model, effective $\mathrm{CD}^{+} \mathrm{T}$ cell immunity was not induced against tumors following intradermal administration of vaccine. In healthy K14-VEGFR3-Ig mice, systemic $\mathrm{T}$ cell responses to dermal vaccination were robust as measured by IFN- $\gamma$ production by $\mathrm{CD}^{+} \mathrm{T}$ cells in spleens 21 days after vac- cination when compared with WT littermates (8). T cell kinetics were delayed, however, with activation primarily occurring in the spleens rather than dLNs. This is consistent with $\mathrm{T}$ cell responses

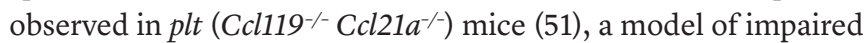
DC trafficking from skin due to a deficiency of homing CCR7 ligands, and $\mathrm{C} c \mathrm{r}^{-1-}$ mice (52). Again, in plt mice, spleens appear to be the main site of activation, as splenectomized mice fail to mount $\mathrm{T}$ cell responses to immunization (51). In our model, we did not observe significant levels of circulating antigen-specific T cells at time of sacrifice, indicating that at least during the experiment we did not generate sufficient adaptive immune responses against implanted, cutaneous tumors. Our observation of decreased fluid drainage and cellular egress to dLNs in K14-VEGFR3-Ig mice supports the model that antitumor $\mathrm{CD}^{+} \mathrm{T}$ cells are primed in the dLN rather than systemically or within tumor microenvironments. While soluble protein and adjuvants might gain access to circulation, implanted tumor cells and associated debris would be restricted to cutaneous tissue and drained either actively or passively to the dLN. As a consequence, adaptive immunity against tumors may be impaired in the absence of lymphatic vessel communication with dLNs. These results corroborate recent work in another model of lymphatic dysfunction in which the Kaposi's sarcoma-associated herpesvirus latent-cycle gene, $k$-cyclin, is expressed under the control of the VEGFR-3 promoter (53). In these mice, antigen presentation in dLNs was impaired and CD8 ${ }^{+}$ T cells demonstrated suppressed cytotoxicity (54). This highlights the important role of dLNs in antitumor immunity $(22,43)$.

Potent adaptive immunity drives compensatory regulatory mechanisms in tissue that impair therapeutic efficacy (40). In the absence of induced adaptive immunity we asked whether mechanisms of immune suppression typically associated with this tumor model were also affected. While we were able to detect inflammatory monocytes and Treg cells in spleen, 2 cell types associated with $\mathrm{CD}^{+} \mathrm{T}$ cell suppression, they were completely absent from tumor microenvironments in K14-VEGFR3-Ig mice. As a result, adoptive $\mathrm{T}$ cell transfer therapy, which is already quite effective in our model, was more efficacious in tumor microenvironments lacking dermal lymphatic vessels. Importantly, we demonstrate enhanced functionality of effector $\mathrm{T}$ cells within tumor microenvironments. In this study, we modeled impaired inflammation and immunity in the absence of dermal lymphatic vessels, thus mimicking patient subsets without detectable systemic immunity. However, patients can also present with detectable peripheral immune responses but still exhibit impaired lymphocyte infiltration within the tumor microenvironment (55). Consequently, additional mechanisms are at play in the clinical setting that limit local antitumor immunity including, for example, impairment of lymphocyte homing due to changes in the tumor-associated vasculature (44) as well as local dysfunction and exhaustion of infiltrating lymphocytes (56). Furthermore, the observation of both inflamed and noninflamed lesions within a single patient, with a single systemic immune response (55), supports the hypothesis that the distinct tumor microenvironments of each lesion as well as tumor cell heterogeneity (29) may act as local regulators of lymphocyte homing, retention, and function. Regional differences in lymphatic vessel function, for example preexisting lymphatic vessel dysfunction and decreased lymphatic vessel density 
in regions of chronic sun exposure and severe solar elastosis (57), may be one mechanism distinguishing inflamed and noninflamed lesions. The specific role of lymphatic vessels and their associated drainage in regulating regional differences in cutaneous immune responses remains an open and exciting question.

Our understanding of the role of lymphatic vessels in the induction and resolution of an antitumor immune response is clearly still in its infancy. While we recently demonstrated an immunosuppressive role for lymphatic vessels in murine melanoma (16), with these current data we also suggest that lymphatic function contributes to local inflammation and induction of antitumor adaptive immune responses. While patients do not present with a complete absence of dermal lymphatic vessels, our transcriptional analysis of human tumors along with the wealth of histological lymphatic vessel density analyses indicates a spectrum of lymphatic vessel involvement, which may correlate with the heterogeneity in immune infiltrate also observed clinically. As a consequence, we propose that the contribution of the lymphatic vasculature and its functional drainage to local immunity may be both a prognostic and targetable feature of tumor microenvironments.

\section{Methods}

TCGA analysis and LS. Level 4 gene expression data were downloaded for metastatic skin cutaneous melanoma from TCGA, which were processed by the Broad Institute's TCGA workgroup (release date 20130523), with no further selection. Of the 266 samples, 212 were from regional sites ( 160 from LNs and 52 from skin or other soft tissue) and 35 were from distant sites (30). The RNA-Seq level 4 gene expression data contain upper quartile-normalized and log2-transformed RSEM (RNA-Seq by expectation maximization) values summarized at the gene level (58). The tumor staging had been performed by the TCGA consortium and every patient used in the study had metastatic disease at the time of biopsy (stage II-IV). All available metastatic cutaneous melanoma samples were analyzed and segregated into 3 groups representing low, mid, and high degrees of lymphatic involvement within the tumor according to their LS (29). To determine LS for each sample, expression levels of the 2 lymphatic markers PDPN and LYVE1, which correlated strongly with each other as well as with the expression levels of $V E G F C$, were first each normalized to their mean expression levels by subtracting the average value and dividing by the $\mathrm{SD}$, such that the average value of the normalized data was zero and the SD was 1 . Then, the average normalized values of PDPN, LYVE1, and $V E G F C$ were added together to form the LS. In this way, the average LS score was zero, and negative values represented below-average lymphatic involvement. Finally, we grouped the patients into $\mathrm{LS}^{\text {mid }}$ (representing LS values within $2 / 3$ of the SD around the mean), LS hi $(>$ mean $+2 / 3 \mathrm{SD})$, and $\mathrm{LS}^{\mathrm{lo}}(<$ mean $-2 / 3 \mathrm{SD}) ; 50 \%$ of patients were $\mathrm{LS}^{\text {mid }}$, while $25 \%$ fell into each of the other 2 groups.

Mice and tumor transplantation. K14-VEGFR3-Ig male mice described elsewhere (33) were maintained on a C57Bl/6 background and crossbred with WT C57Bl/6 female mice. Ten- to twelve-week-old K14-VEGFR3-Ig mice and WT littermates of both sexes were used in all experiments. Chy mice, described previously (36), were obtained from MRC-Harwell distributing mice on behalf of the European Mouse Mutant Archive (C3H1O1H-Flt $4<\mathrm{Chy}>/ \mathrm{H}$ mice, repository number EM:00068), and were maintained on a C3H background. TCR-Tg OT-I mice were purchased from Harlan Laboratories and maintained in house. WT C57Bl/6 mice were purchased from Harlan Laboratories. Animals were housed in pathogen-free facilities. B16F10 (ATCC), B16F10.OVA (gift of Bertrand Huard, University of Geneva, Geneva, Switzerland) and C3HBA (NCI-Frederick Cancer DCT Tumor Repository) cells were grown at $37^{\circ} \mathrm{C}$ in $5 \% \mathrm{CO}_{2}$ in Dulbecco's Modified Eagle's Medium (DMEM, Sigma-Aldrich), supplemented with nonessential amino acids and 10\% fetal bovine serum, $100 \mathrm{U} /$ $\mathrm{ml}$ penicillin, $100 \mu \mathrm{g} / \mathrm{ml}$ streptomycin, and $400 \mu \mathrm{M} \mathrm{L}$-glutamine (all from Lonza). Cells were detached with trypsin-EDTA (Sigma-Aldrich) and passaged every 3 days. B16F10 cells $\left(0.5 \times 10^{6}\right)$ in $50-100 \mu$ l saline were inoculated intradermally and dorsolaterally. Tumor growth was measured daily using calipers. For intradermal ear injections, animals were anesthetized with $1 \%$ isoflurane in combination with $\mathrm{O}_{2}$ and $\mathrm{N}_{2}$ and $20 \mu \mathrm{l}$ of cell slurry was injected into the dermis of the ear. In some mice, $500 \mu \mathrm{g}$ anti-VEGFR-3 neutralizing antibody (mF4-31C1, Eli Lilly and Company) was administered on days 0,3 , and 6 after tumor inoculation. For metastasis experiments, B16F10 cells $\left(5 \times 10^{5}\right)$ were introduced via tail vein injection and animals were sacrificed 14 days later. In some cases, 6 hours prior to sacrifice, mice were given $250 \mu \mathrm{g}$ brefeldin A i.p. in preparation for intracellular cytokine staining.

Quantification of metastasis. For the quantification of metastatic melanomas, lungs isolated from mice after s.c. or i.v. injection of B16F10 melanoma cells were paraffin embedded and microsectioned at 3 different planes $(\sim 350 \mu \mathrm{m}$ apart) and stained with hematoxylin and eosin. Histological slides ( 3 per mouse) were then inspected blindly for metastatic melanomas. The quantification of tumors in each image is relative to the total tissue area and is presented as a percentage: tumor-to-lung ratio $=$ tumor area $/$ total lung area $\times 100$.

Fluid drainage and DC trafficking. Either $20 \mu \mathrm{l} 0.5-\mu \mathrm{m}$ FITC-conjugated latex microspheres (Polysciences, diluted 1:25 in sterile saline) or $20 \mu \mathrm{l}$ FITC-dextran was injected into the tumor with a 30-gauge needle. After 30 minutes (for dextran) or 24 hours (for microspheres), tumors and LNs (brachial) were harvested. For dextran drainage, LNs were homogenized and fluorescence quantified by plate reader (Safire ${ }^{2}$, TECAN). For DC trafficking, single-cell suspensions were prepared and analyzed by flow cytometry for $\mathrm{CD} 11 \mathrm{c}^{+} \mathrm{MHCII}{ }^{+} \mathrm{FITC}^{+} \mathrm{DCs}$.

Prophylactic vaccination. Mice received $10 \mu \mathrm{g}$ LPS as a negative control or $10 \mu \mathrm{g}$ LPS plus $10 \mu \mathrm{g}$ OVA (grade IV, Sigma-Aldrich) in 2 intradermal doses of $25 \mu \mathrm{l}$ per foreleg. Ten days later, $5 \times 10^{5} \mathrm{~B} 16 \mathrm{~F} 10$. OVA or OVA/VEGF- $\mathrm{C}^{+}$tumor cells in $50 \mu$ were inoculated intradermally and dorsolaterally, and a vaccine boost (identical to the first) was administered again on day 15.

Adoptive T cell transfer. Splenic CD $8^{+} \mathrm{T}$ cells and CD11 $\mathrm{c}^{+} \mathrm{DC}$ were isolated by positive magnetic cell sorting (Miltenyi Biotech). Purified CD8 ${ }^{+}$OT-I and splenic DCs were cocultured at a ratio of 10:1, supplemented with $1 \mathrm{nM}$ SIINFEKL (GenScript) and $10 \mathrm{U} / \mathrm{ml}$ IL-2 (Roche). Cells were collected after 4 days and injected i.v. into tumor-bearing mice $\left(1 \times 10^{6}\right.$ cells in $\left.200 \mu \mathrm{l}\right)$.

Immunohistochemistry. Tumor samples were fixed in $4 \%$ paraformaldehyde, paraffin-embedded, and cut into $4-\mu \mathrm{m}$ sections. For immunolabeling, paraffin-embedded tissue sections were deparaffinized and rehydrated before antigen retrieval at $98^{\circ} \mathrm{C}$ for 1 hour in 0.01 $\mathrm{M}$ citrate buffer ( $\mathrm{pH}$ 6.0). Antigen retrieval for $\mathrm{F} 4 / 80$ staining was performed with Proteinase K (Dako) treatment according to the manufacturer's instructions. After blocking with diluted serum from the secondary antibody host for 30 minutes, the slides were incubated overnight $\left(4^{\circ} \mathrm{C}\right)$ with the primary antibody. Endogenous peroxidase activity was 
blocked for 20 minutes with 3\% hydrogen peroxide (Sigma-Aldrich) and a biotinylated anti-rat, anti-rabbit, anti-hamster, or anti-goat secondary antibody (Vector Laboratories) was applied for 45 minutes. The antigen-antibody complex reaction was augmented with avidin-biotin-peroxidase for 45 minutes according to the manufacturer's instructions (Vectastain ABC Kit, Vector) and stained for 1-10 minutes with diaminobenzidine tetrahydrochloride (DAB, Vector). The sections were then counterstained with hematoxylin (Thermo Scientific), dehydrated, and mounted with Entellan (Electron Microscopy Services). Primary antibodies used were: rabbit anti-LYVE1 (1:100, catalog ab14917, Abcam), rabbit anti-CD11b (1:200, catalog ab75476, Abcam), rat anti-F4/80 (1:50, catalog ab6640-200, Abcam), rat anti-MHCII (I-A/I-E) (1:100, catalog 11-5322-82, eBioscience), hamster anti-CD3e (1:100, cata$\log 550277$, Abcam), rabbit anti-alpha SMA (1:100, catalog ab5694, Abcam), goat anti-CD31 (1:100, catalog sc1506, Santa Cruz Biotechnology), and rat anti-CD45 (1:250, clone 30-F11, BD Pharmingen).

Immunohistochemical staining of human tissue. Human tissue was obtained by surgical excision from a patient with primary melanoma, and used for research upon informed consent at the Ludwig Institute for Cancer Research (Canton of Vaud University Hospital) as a part of a phase I vaccination trial (LUD 00-018 study, ClinicalTrials.gov Identifier NCT00112229). The tissue was formalin fixed and paraffin embedded and 4- $\mu \mathrm{m}$ sections were prepared. Antigen retrieval for melan-A and PROX-1 staining was performed with Tris-EDTA ( $\mathrm{pH}$ 9) for 15 minutes. Primary antibodies used were: mouse anti-human melan-A (1:50, clone A103, Dako) and mouse anti-human PROX1 (1:100, catalog AF2727-SP, R\&D Systems). Alexa Fluor-labeled secondary antibodies were incubated for 1 hour at room temperature (1:400, Invitrogen). Sections were counterstained with DAPI (Lucerna Chem AG) and coverslipped with fluorescence mounting medium (S3023, DAKO).

Picrosirius red staining. Paraffin-embedded sections were deparaffinized and hydrated in xylene and ethanol. The slides were then stained with picrosirius red solution composed of picric acid and Direct Red 80 (both Sigma-Aldrich) according to the manufacturer's instructions and dehydrated in ethanol and xylene. Sections were washed in 2 changes of acidified water after picrosirius red staining.

Western blot and cytokine array. Tumor tissues were homogenized and lysed in a custom-made total protein lysis buffer $(50 \mathrm{mM}$ Tris $\mathrm{HCl}$, pH 7.5, 150 mM NaCl, 0.1\% SDS, 1\% deoxycholate, 1\% Triton X-100) containing protease and phosphatase inhibitors (Roche). Protein concentration was measured using a bicinchoninic acid (BCA) assay (Pierce) and $30 \mu \mathrm{g}$ protein was loaded per lane for all immunoblots. The protein lysates were resolved using NuPAGE Novex 4\%-12\% Bis-Tris Gels (Invitrogen). Protein transfer was assessed by Ponceau $\mathrm{S}$ solution (Sigma-Aldrich), and then membranes were blocked with $5 \%$ fat-free dry milk for 1 hour. Immobilized antibody was detected using the horseradish peroxidase-conjugated secondary antibodies and SuperSignal West Pico Chemiluminescent Substrate (Thermo Scientific). The immune reaction was visualized with an LAS-3000 imaging system (FujiFilm). Primary antibodies used were: rat antiGM-CSF (1:500, catalog ab13789-100, Abcam), rabbit anti-MCP-1 (1:2,000; catalog NB110-2000; Novus Biologicals), rabbit anti-IFN- $\gamma$ (1:500, catalog AAM29, AbD Serotec), rabbit anti-TNF- $\alpha$ (1:100, catalog ACC-250844, BioSite), rabbit anti-NOS2 (1:1,000; catalog 2982S; Cell Signaling Technology), goat anti-IL-1 3 (1:500, AF-401-NA, R\&D Systems), rat anti-IL-10 (1:500, catalog ab33471, Abcam), rabbit anti-
VEGF (1:500, catalog ab46154, Abcam), goat anti-MCSF (1:500, catalog af416-sp, Novus Biologicals), rabbit anti-TGF- $\beta$ (1:500, catalog AB-100NA, R\&D Systems) and rabbit anti- $\beta$ actin (1:500, catalog ab8227, Abcam).

The relative levels of 22 different cytokines and chemokines in tumor lysates were evaluated using a Mouse Cytokine Antibody Array Kit (ab133993, Abcam) according to the manufacturer's protocol. Immunoblot images were captured and visualized using the LAS3000 imaging system and spot intensity was analyzed using Image J v1.49 software (NIH). The values are shown as relative intensity of expression compared with an internal control (biotinylated IgG). Cytokines detected by the antibody array kit were: G-CSF, GM-CSF, IL-2, IL-3, IL-4, IL-5, IL-6, IL-9, IL-10, IL-12 p40/p70, IL-12p70, IL-13, IL-17, IFN- $\gamma$, MCP-1, MCP-5, RANTES, stem cell factor, soluble TNF receptor I (TNF-RI), TNF- $\alpha$, thrombopoietin, VEGF.

Flow cytometry. Tumors and LNs (draining and nondraining) were incubated in collagenase D (1 mg/ml in HBSS with 2\% FBS) for 1 hour at $37^{\circ} \mathrm{C}$ followed by $100 \mathrm{mM}$ EDTA and then pushed through a $70-\mu \mathrm{m}$ strainer to create a single-cell suspension. The following anti-mouse antibodies were used for flow cytometry: CD45-APC, CD45-Pacific blue, or biotinylated CD45 (clone 30-F11); CD3e-Pacific blue (clone 17A2) or CD3ع-PerCPCy5.5 (clone 145-2C11); CD4-PECy7 or CD4-PE (clone GK1.5); CD8 $\alpha$-PECy7 or CD8 $\alpha$-APC-Alexa 780 (clone 53-6.7); F4/80-PE (clone BM8); CD25-FITC (clone 3C7); FoxP3-PerCPCy5.5 (clone FJK-16s); CD11c-Alexa 647 (clone N418); MHCII-FITC (clone M5/114.15.2); biotinylated Gr1 (clone RB6-8C5); CD45.2-Pacific blue (clone 104); IFN- $\gamma$-APC (clone XMG1.2); and CD11b-PECy7 (clone $\mathrm{M} 1 / 70$ ) (all from eBioscience). Pentamer staining for Trp2 H-2Kb-PE (ProImmune) and H-2Kb-SIINFEKL-PE (ProImmune) was performed according to the manufacturers' guidelines. Cell viability was determined using LIVE/DEAD Fixable Dead Cell Stain Kits or propidium iodide (both Invitrogen).

Statistics. Unless stated differently, statistical significance was analyzed by unpaired, 2-tailed Student's $t$ test using Prism software (GraphPad Inc.); $P<0.05$ was considered to be statistically significant. Mann-Whitney $U$ tests were performed on in vivo data unless the data were confirmed to fulfill the criteria of normal distribution and equal variance, after which $t$ tests and ANOVA were performed. Regression analysis was performed to compare slopes of tumor growth curves (GraphPad Inc.).

Study approval. All animal experiments were conducted in accordance with the regulations of the Norwegian State Commission for Laboratory Animals and the Swiss Animal Protection Act, which are consistent with the European Convention for the Protection of Vertebrate Animals used for Experimental and Other Scientific Purposes and Council of Europe (ETS 123), and with approval from the AAALAC International accredited Animal Care and Use Program at University of Bergen and the Cantonal Veterinary Office of Canton de Vaud, Switzerland.

\section{Author contributions}

AWL, MW, HW, and MAS conceived the ideas and designed the experiments. AWL, MW, MF, ESS, and MAB performed experiments and analyzed the data. SS and TFG provided normalized TCGA dataset. KA provided the K14-VEGFR3-Ig model. HPE aided data acquisition and interpretation. AWL, MW, HW, and MAS wrote the paper. 


\section{Acknowledgments}

The authors are grateful to Yassin Ben Saida for technical assistance, Bronek Pytowski (Eli Lilly and Company) for the mF431C antibody, and to Daniel Speiser (The Ludwig Institute for Cancer Research, Canton deVaud University Hospital, Lausanne, Switzerland) for human primary melanoma samples. This work was funded in part by grants from the European Research Council (grant AdG-323053 to MAS), the Swiss National Science Foundation (grant 31-153471 to MAS), the Swiss Cancer League (grant KFS 3312-08-2013 to MAS), the Western Norway Regional Health Authority (grant 911532 to MW and 911888 to
HW), Rakel and Otto Kristian Bruun's Fund (to HW), and from The Research Council of Norway (grant 222278 to HW). SS is supported by the Irvington Postdoctoral Fellowship by the Cancer Research Institute.

Address correspondence to: Helge Wiig, University of Bergen, Jonas Lies vei 91, Postboks 7804, 5020 Bergen, Norway. Phone: 47.55.58.63.87; E-mail: helge.wiig@uib.no. Or to: Melody A. Swartz, University of Chicago, Eckhardt Research Center, Room 379, 5640 South Ellis Avenue, Chicago, Illinois 60637, USA. Phone: 773.702.0452; E-mail: melodyswartz@uchicago.edu.
1. Pasquali S, van der Ploeg AP, Mocellin S, Stretch JR, Thompson JF, Scolyer RA. Lymphatic biomarkers in primary melanomas as predictors of regional lymph node metastasis and patient outcomes. Pigment Cell Melanoma Res. 2013;26(3):326-337.

2. Karaman S, Detmar M. Mechanisms of lymphatic metastasis. J Clin Invest. 2014;124(3):922-928.

3. Platt AM, Randolph GJ. Dendritic cell migration through the lymphatic vasculature to lymph nodes. Adv Immunol. 2013;120:51-68.

4. Swartz MA, Lund AW. Lymphatic and interstitial flow in the tumour microenvironment: linking mechanobiology with immunity. Nat Rev Cancer. 2012;12(3):210-219.

5. Wiig H, Swartz MA. Interstitial fluid and lymph formation and transport: physiological regulation and roles in inflammation and cancer. Physiol Rev. 2012;92(3):1005-1060.

6. Platt AM, et al. Normal dendritic cell mobilization to lymph nodes under conditions of severe lymphatic hypoplasia. J Immunol. 2013;190(9):4608-4620.

7. Clement CC, et al. An expanded self-antigen peptidome is carried by the human lymph as compared to the plasma. PLoS One. 2010;5(3):e9863.

8. Thomas SN, et al. Impaired humoral immunity and tolerance in K14-VEGFR-3-Ig mice that lack dermal lymphatic drainage. JImmunol. 2012;189(5):2181-2190.

9. Iolyeva M, et al. Interleukin-7 is produced by afferent lymphatic vessels and supports lymphatic drainage. Blood. 2013;122(13):2271-2281.

10. Podgrabinska S, et al. Inflamed lymphatic endothelium suppresses dendritic cell maturation and function via Mac-1/ICAM-1-dependent mechanism. J Immunol. 2009;183(3):1767-1779.

11. Miteva DO, Rutkowski JM, Dixon JB, Kilarski W, Shields JD, Swartz MA. Transmural flow modulates cell and fluid transport functions of lymphatic endothelium. Circ Res. 2010;106(5):920-931.

12. Huggenberger R, Ullmann S, Proulx ST, Pytowski B, Alitalo K, Detmar M. Stimulation of lymphangiogenesis via VEGFR-3 inhibits chronic skin inflammation. JExp Med. 2010;207(10):2255-2269.

13. D'Alessio S, et al. VEGF-C-dependent stimulation of lymphatic function ameliorates experimental inflammatory bowel disease. J Clin Invest. 2014;124(9):3863-3878.

14. Cohen JN, et al. Lymph node-resident lymphatic endothelial cells mediate peripheral tolerance via Aire-independent direct antigen presentation. JExp Med. 2010;207(4):681-688.
15. Hirosue S, et al. Steady-state antigen scavenging, cross-presentation, and $\mathrm{CD}^{+} \mathrm{T}$ cell priming: a new role for lymphatic endothelial cells. J Immunol. 2014;192(11):5002-5011.

16. Lund AW, et al. VEGF-C promotes immune tolerance in B16 melanomas and cross-presentation of tumor antigen by lymph node lymphatics. Cell Rep. 2012;1(3):191-199.

17. Alitalo K. The lymphatic vasculature in disease. Nat Med. 2011;17(11):1371-1380.

18. Hos D, Cursiefen C. Lymphatic vessels in the development of tissue and organ rejection. $A d v$ Anat Embryol Cell Biol. 2014;214:119-141.

19. Lund AW, Medler TR, Leachman SA, Coussens LM. Lymphatic vessels, inflammation, and immunity in skin cancer. Cancer Discov. 2016;6(1):22-35.

20. Ruffell B, Affara NI, Coussens LM. Differential macrophage programming in the tumor microenvironment. Trends Immunol. 2012;33(3):119-126.

21. Crusz SM, Balkwill FR. Inflammation and cancer: advances and new agents. Nat Rev Clin Oncol. 2015;12(10):584-596.

22. Thomas SN, Vokali E, Lund AW, Hubbell JA, Swartz MA. Targeting the tumor-draining lymph node with adjuvanted nanoparticles reshapes the anti-tumor immune response. Biomaterials. 2014;35(2):814-824.

23. Speiser DE, et al. Memory and effector CD8 $\mathrm{T}$-cell responses after nanoparticle vaccination of melanoma patients. JImmunother. 2010;33(8):848-858.

24. Hodi FS, et al. Improved survival with ipilimumab in patients with metastatic melanoma. $N$ Engl J Med. 2010;363(8):711-723.

25. Topalian SL, et al. Safety, activity, and immune correlates of anti-PD-1 antibody in cancer. $N$ Engl JMed. 2012;366(26):2443-2454.

26. Harlin $\mathrm{H}$, et al. Chemokine expression in melanoma metastases associated with $\mathrm{CD} 8^{+} \mathrm{T}$-cell recruitment. Cancer Res. 2009;69(7):3077-3085.

27. Ji RR, et al. An immune-active tumor microenvironment favors clinical response to ipilimumab. Cancer Immunol Immunother. 2012;61(7):1019-1031.

28. Tumeh PC, et al. PD-1 blockade induces responses by inhibiting adaptive immune resistance. Nature. 2014;515(7528):568-571.

29. Spranger S, Bao R, Gajewski TF. Melanomaintrinsic $\beta$-catenin signalling prevents anti-tumour immunity. Nature. 2015;523(7559):231-235.

30. Cancer Genome Atlas Network. Genomic classification of cutaneous melanoma. Cell. 2015;161(7):1681-1696.
31. Harvey NL, Gordon EJ. Deciphering the roles of macrophages in developmental and inflammation stimulated lymphangiogenesis. Vasc Cell. 2012;4(1):15

32. Shields JD, Kourtis IC, Tomei AA, Roberts JM, Swartz MA. Induction of lymphoidlike stroma and immune escape by tumors that express the chemokine CCL21. Science. 2010;328(5979):749-752.

33. Mäkinen T, et al. Inhibition of lymphangiogenesis with resulting lymphedema in transgenic mice expressing soluble VEGF receptor-3. Nat Med. 2001;7(2):199-205.

34. Alitalo AK, et al. VEGF-C and VEGF-D blockade inhibits inflammatory skin carcinogenesis. Cancer Res. 2013;73(14):4212-4221.

35. Randolph GJ, Angeli V, Swartz MA. Dendritic-cell trafficking to lymph nodes through lymphatic vessels. Nat Rev Immunol. 2005;5(8):617-628.

36. Karkkainen MJ, et al. A model for gene therapy of human hereditary lymphedema. Proc Natl Acad Sci U S A. 2001;98(22):12677-12682.

37. Rutkowski JM, Markhus CE, Gyenge CC, Alitalo K, Wiig H, Swartz MA. Dermal collagen and lipid deposition correlate with tissue swelling and hydraulic conductivity in murine primary lymphedema. Am J Pathol. 2010;176(3):1122-1129.

38. Alexandrov LB, et al. Signatures of mutational processes in human cancer. Nature. 2013;500(7463):415-421.

39. Drake CG, Lipson EJ, Brahmer JR. Breathing new life into immunotherapy: review of melanoma, lung and kidney cancer. Nat Rev Clin Oncol. 2014;11(1):24-37.

40. Gajewski TF, et al. Cancer immunotherapy strategies based on overcoming barriers within the tumor microenvironment. Curr Opin Immunol. 2013;25(2):268-276.

41. Peranzoni E, et al. Myeloid-derived suppressor cell heterogeneity and subset definition. Curr Opin Immunol. 2010;22(2):238-244.

42. Talmadge JE. Pathways mediating the expansion and immunosuppressive activity of myeloid-derived suppressor cells and their relevance to cancer therapy. Clin Cancer Res. 2007;13(18 Pt 1):5243-5248.

43. Jeanbart L, et al. Enhancing efficacy of anticancer vaccines by targeted delivery to tumor-draining lymph nodes. Cancer Immunol Res. 2014;2(5):436-447.

44. Motz GT, Coukos G. The parallel lives of angiogenesis and immunosuppression: cancer and other tales. Nat Rev Immunol. 2011;11(10):702-711. 45. Xu Y, et al. Neuropilin-2 mediates VEGF-C- 


\section{RESEARCH ARTICLE}

induced lymphatic sprouting together with VEGFR3. J Cell Biol. 2010;188(1):115-130.

46. Landry CS, et al. Resection of at-risk mesenteric lymph nodes is associated with improved survival in patients with small bowel neuroendocrine tumors. World J Surg. 2013;37(7):1695-1700.

47. Glover AR, Allan CP, Wilkinson MJ, Strauss DC, Thomas JM, Hayes AJ. Outcomes of routine ilioinguinal lymph node dissection for palpable inguinal melanoma nodal metastasis. Br J Surg. 2014;101(7):811-819.

48. Kitamura T, Qian BZ, Pollard JW. Immune cell promotion of metastasis. Nat Rev Immunol. 2015;15(2):73-86.

49. Lin EY, Nguyen AV, Russell RG, Pollard JW. Colony-stimulating factor 1 promotes progression of mammary tumors to malignancy. J Exp Med. 2001;193(6):727-740.
50. Harney AS, et al. Real-time imaging reveals local, transient vascular permeability, and tumor cell intravasation stimulated by TIE2hi macrophage-derived VEGFA. Cancer Discov. 2015;5(9):932-943.

51. Mori S, Nakano H, Aritomi K, Wang CR, Gunn MD, Kakiuchi T. Mice lacking expression of the chemokines CCL21-ser and CCL19 (plt mice) demonstrate delayed but enhanced $\mathrm{T}$ cell immune responses. J Exp Med. 2001;193(2):207-218.

52. Förster R, et al. CCR7 coordinates the primary immune response by establishing functional microenvironments in secondary lymphoid organs. Cell. 1999;99(1):23-33.

53. Sugaya M, et al. Lymphatic dysfunction in transgenic mice expressing KSHV k-cyclin under the control of the VEGFR-3 promoter. Blood. 2005;105(6):2356-2363.
54. Kimura T, Sugaya M, Oka T, Blauvelt A, Okochi $\mathrm{H}$, Sato S. Lymphatic dysfunction attenuates tumor immunity through impaired antigen presentation. Oncotarget. 2015;6(20):18081-18093.

55. Gajewski TF. The next hurdle in cancer immunotherapy: overcoming the non-T-cell-inflamed tumor microenvironment. Semin Oncol. 2015;42(4):663-671.

56. Baitsch L, et al. Exhaustion of tumor-specific $\mathrm{CD}^{+} \mathrm{T}$ cells in metastases from melanoma patients. J Clin Invest. 2011;121(6):2350-2360.

57. Kajiya K, Kunstfeld R, Detmar M, Chung JH. Reduction of lymphatic vessels in photodamaged human skin. J Dermatol Sci. 2007;47(3):241-243.

58. Li B, Dewey CN. RSEM: accurate transcript quantification from RNA-Seq data with or without a reference genome. BMC Bioinformatics. 2011;12:323. 